J. Korean Math. Soc. 51 (2014), No. 3, pp. 567-592

http://dx.doi.org/10.4134/JKMS.2014.51.3.567

\title{
CONSTRUCTION OF SUBCLASSES OF UNIVALENT HARMONIC MAPPINGS
}

\author{
Sumit NagPal and V. Ravichandran
}

\begin{abstract}
Complex-valued harmonic functions that are univalent and sense-preserving in the open unit disk are widely studied. A new methodology is employed to construct subclasses of univalent harmonic mappings from a given subfamily of univalent analytic functions. The notions of harmonic Alexander operator and harmonic Libera operator are introduced and their properties are investigated.
\end{abstract}

\section{1. introduction}

Let $\mathcal{H}$ denote the class of all complex-valued harmonic functions $f$ in the open unit disk $\mathbb{D}:=\{z \in \mathbb{C}:|z|<1\}$ normalized by $f(0)=0=f_{z}(0)-1=$ $f_{\bar{z}}(0)$. Such functions can be written in the form $f=h+\bar{g}$, where

$$
h(z)=z+\sum_{n=2}^{\infty} a_{n} z^{n} \quad \text { and } \quad g(z)=\sum_{n=2}^{\infty} b_{n} z^{n}
$$

are analytic in $\mathbb{D}$. In 1984, Clunie and Sheil-Small [7] investigated the subclass $\mathcal{S}_{H}^{0}$ of $\mathcal{H}$ consisting of univalent and sense-preserving functions. A function $f=h+\bar{g} \in \mathcal{H}$ is sense-preserving if the Jacobian $J_{f}(z)=\left|h^{\prime}(z)\right|^{2}-\left|g^{\prime}(z)\right|^{2}$ is positive or equivalently $\left|g^{\prime}(z)\right|<\left|h^{\prime}(z)\right|$ in $\mathbb{D}$. The class $\mathcal{S}_{H}^{0}$ is a compact family with respect to the topology of locally uniform convergence. The classical family $\mathcal{S}$ of normalized analytic univalent functions is a subclass of $\mathcal{S}_{H}^{0}$. Let $\mathcal{S}_{H}^{* 0}, \mathcal{K}_{H}^{0}$ and $\mathcal{C}_{H}^{0}$ be the subclasses of $\mathcal{S}_{H}^{0}$ consisting of functions mapping $\mathbb{D}$ onto starlike, convex and close-to-convex domains, respectively, just as $\mathcal{S}^{*}, \mathcal{K}$ and $\mathcal{C}$ are the subclasses of $\mathcal{S}$ mapping $\mathbb{D}$ onto their respective domains.

In [26], we have investigated the properties of functions in the subclass $\mathcal{F}_{H}^{0} \subset$ $\mathcal{C}_{H}^{0}$ defined by the condition $\left|f_{z}(z)-1\right|<1-\left|f_{\bar{z}}(z)\right|$ for all $z \in \mathbb{D}$. This subclass was closely related to the class $\mathcal{F} \subset \mathcal{C}$, introduced by MacGregor [20], consisting

Received September 22, 2013.

2010 Mathematics Subject Classification. Primary 31A05, Secondary 30C45.

Key words and phrases. univalent harmonic mappings, starlike, convex, close-to-convex, coefficient estimates, growth estimate, convolution, convex combination, integral operators.

The research work of the first author is supported by research fellowship from Council of Scientific and Industrial Research (CSIR), New Delhi. 
of analytic functions satisfying $\left|f^{\prime}(z)-1\right|<1$ for $z \in \mathbb{D}$. We [26] proved that a harmonic function $f=h+\bar{g} \in \mathcal{F}_{H}^{0}$ if and only if the analytic functions $h+\epsilon g$ belong to $\mathcal{F}$ for each $|\epsilon|=1$. Using this property, the coefficient estimates, growth results, boundary behavior, convolution properties and sharp bound for radius of convexity and starlikeness for the class $\mathcal{F}_{H}^{0}$ were investigated. This connection between the classes $\mathcal{F}$ and $\mathcal{F}_{H}^{0}$ has motivated to give the following definition which turns out to be a simple but an effective method in construction of subclasses of univalent harmonic mappings from a given subfamily of $\mathcal{S}$.

Definition 1.1. Suppose that $\mathcal{G}$ is a subfamily of $\mathcal{S}$. Denote by $\mathcal{G}_{H}^{0}$ the class consisting of harmonic functions $f=h+\bar{g}$ for which $h+\epsilon g \in \mathcal{G}$ for each $|\epsilon|=1$, $h$ and $g$ being analytic functions in $\mathbb{D}$. We call $\mathcal{G}_{H}^{0}$ the harmonic analogue of $\mathcal{G}$ and write $\mathcal{G} \triangleright \mathcal{G}_{H}^{0}$.

By Definition 1.1, it readily follows that $\mathcal{F} \triangleright \mathcal{F}_{H}^{0}$. If $\mathcal{G}_{H}^{0}$ is the harmonic analogue of $\mathcal{G} \subset \mathcal{S}$, then it is easy to see that $\mathcal{G} \subset \mathcal{G}_{H}^{0}$. Further properties of the harmonic analogue $\mathcal{G}_{H}^{0}$ for a subfamily $\mathcal{G} \subset \mathcal{S}$ are investigated in Section 2 . In Section 3, the harmonic analogues of some well-known subclasses of $\mathcal{S}$ are determined and their properties are discussed.

Let $\mathcal{A}$ be the subclass of $\mathcal{H}$ consisting of normalized analytic functions. Let $\Lambda: \mathcal{A} \rightarrow \mathcal{A}$ be the Alexander integral operator [1] defined by

$$
\Lambda[f](z)=\int_{0}^{z} \frac{f(t)}{t} d t .
$$

Krzyż and Lewandowski [16] constructed an example to show that $\Lambda$ does not carry $\mathcal{S}$ into $\mathcal{S}$. Another familiar integral operator $\Theta: \mathcal{A} \rightarrow \mathcal{A}$ is the Libera operator [18] defined by

$$
\Theta[f](z)=\frac{2}{z} \int_{0}^{z} f(t) d t .
$$

Even this operator does not preserve univalence. Campbell and Singh [4] gave examples of univalent functions which the operator $\Theta$ takes to non-univalent functions. However, these two operators preserve certain subclasses of univalent functions. In the last section of this paper, two notions of harmonic Alexander operators $\Lambda_{H}^{+}, \Lambda_{H}^{-}: \mathcal{H} \rightarrow \mathcal{H}$ and a notion of harmonic Libera operator $\Theta_{H}$ : $\mathcal{H} \rightarrow \mathcal{H}$ are introduced and their properties are investigated.

\section{Some properties of harmonic analogue $\mathcal{G}_{H}^{0}$}

In this section, we will investigate the properties of the harmonic analogue $\mathcal{G}_{H}^{0}$ for subfamily $\mathcal{G} \subset \mathcal{S}$. For this, the notion of stable harmonic mappings introduced by Hernández and Martín in [15] is needed. A sense-preserving harmonic mapping $f=h+\bar{g}$ is said to be stable univalent (resp. stable starlike, stable convex and stable close-to-convex) if all the mappings $f_{\lambda}=h+\lambda \bar{g}$ with $|\lambda|=1$ are univalent (resp. starlike, convex and close-to-convex) in $\mathbb{D}$. The following result was proved in [15]. 
Lemma 2.1. A sense-preserving harmonic mapping $f=h+\bar{g}$ is stable univalent (resp. stable starlike, stable convex and stable close-to-convex) if and only if the analytic functions $F_{\lambda}=h+\lambda g$ are univalent (resp. starlike, convex and close-to-convex) in $\mathbb{D}$ for each $|\lambda|=1$.

Let $\mathcal{S S}_{H}^{0}, \mathcal{S S}_{H}^{* 0}, \mathcal{S} \mathcal{K}_{H}^{0}$ and $\mathcal{S C}_{H}^{0}$ be subclasses of $\mathcal{S}_{H}^{0}$ consisting of stable univalent, stable starlike, stable convex and stable close-to-convex mappings, respectively. Then $\mathcal{S}^{*} \subset \mathcal{S S}_{H}^{* 0} \subset \mathcal{S}_{H}^{* 0}, \mathcal{K} \subset \mathcal{S K}_{H}^{0} \subset \mathcal{K}_{H}^{0}$ and $\mathcal{C} \subset \mathcal{S C}_{H}^{0} \subset \mathcal{C}_{H}^{0}$. Moreover $\mathcal{S K}_{H}^{0} \subset \mathcal{S} \mathcal{S}_{H}^{* 0} \subset \mathcal{S C}_{H}^{0} \subset \mathcal{S S}_{H}^{0}$. In view of Definition 1.1 and Lemma 2.1 , it follows that $\mathcal{S S}_{H}^{0}, \mathcal{S S}_{H}^{* 0}, \mathcal{S K}_{H}^{0}$ and $\mathcal{S C}_{H}^{0}$ are harmonic analogues of $\mathcal{S}$, $\mathcal{S}^{*}, \mathcal{K}$ and $\mathcal{C}$, respectively.

The first theorem is quite simple but a useful tool in the investigation of results regarding the harmonic analogue $\mathcal{G}_{H}^{0}$ for a subfamily $\mathcal{G} \subset \mathcal{S}$.

Theorem 2.2. Suppose that $\mathcal{G} \subset \mathcal{S}$ and $\mathcal{G} \triangleright \mathcal{G}_{H}^{0}$. Then

(i) $\mathcal{G}_{H}^{0} \subset \mathcal{S S}_{H}^{0}$;

(ii) If $f \in \mathcal{S} \cap \mathcal{G}_{H}^{0}$, then $f \in \mathcal{G}$;

(iii) If $f=h+\bar{g} \in \mathcal{G}_{H}^{0}$, then the harmonic mappings $f_{\lambda}=h+\lambda \bar{g} \in \mathcal{G}_{H}^{0}$ for each $|\lambda|=1$

(iv) If $\mathcal{J} \subset \mathcal{G}$, then $\mathcal{J}_{H}^{0} \subset \mathcal{G}_{H}^{0}$ where $\mathcal{J}_{H}^{0}$ is the harmonic analogue of $\mathcal{J}$.

Proof. Let $f=h+\bar{g} \in \mathcal{G}_{H}^{0}$. Then $h+\epsilon g \in \mathcal{G}$ for each $|\epsilon|=1$ which imply that $h(0)=g(0)=h^{\prime}(0)-1=g^{\prime}(0)=0$ using the normalization of functions in $\mathcal{G}$. Also, since $h+\epsilon g$ is univalent, $(h+\epsilon g)^{\prime} \neq 0$ in $\mathbb{D}$ for each $|\epsilon|=1$. This imply that the Jacobian $J_{f}(z) \neq 0$ for all $z \in \mathbb{D}$ and since $J_{f}(0)=1>0, f$ is sense-preserving in $\mathbb{D}$. By Lemma 2.1, it follows that $f \in \mathcal{S S}_{H}^{0}$. This proves (i). The part (ii) follows immediately from Definition 1.1. For the proof of (iii), let $f=h+\bar{g} \in \mathcal{G}_{H}^{0}$ and $|\lambda|=1$. Then it is easy to see that $h+\bar{\lambda} \epsilon g \in \mathcal{G}$ for each $|\epsilon|=1$ and so $h+\lambda \bar{g} \in \mathcal{G}_{H}^{0}$. To prove (iv), let $f=h+\bar{g} \in \mathcal{J}_{H}^{0}$. As $\mathcal{J} \triangleright \mathcal{J}_{H}^{0}$, $h+\epsilon g \in \mathcal{J}$ for each $|\epsilon|=1$. Since $\mathcal{J} \subset \mathcal{G}$ we have $h+\epsilon g \in \mathcal{G}$ for each $|\epsilon|=1$ which shows that $f \in \mathcal{G}_{H}^{0}$. This completes the proof of the theorem.

Theorem 2.2(ii) conveys that every analytic univalent function in $\mathcal{G}_{H}^{0}$ is a member of $\mathcal{G}$. Since the members of $\mathcal{G}_{H}^{0}$ are stable univalent by Theorem 2.2(i), we have the following corollary which follows by [15, Theorem 7.1].

Corollary 2.3. Suppose that $\mathcal{G} \subset \mathcal{S}$ and $\mathcal{G} \triangleright \mathcal{G}_{H}^{0}$. If $f=h+\bar{g} \in \mathcal{G}_{H}^{0}$, then the analytic mappings $F_{\mu}=h+\mu$ are univalent in $\mathbb{D}$ for each $|\mu| \leq 1$. In particular, $h$ is univalent.

Recall that convexity and starlikeness are hereditary properties for conformal mappings and they do not extend to harmonic mappings (see [10]). Chuaqui, Duren and Osgood [6] introduced the notion of fully starlike and fully convex functions that do inherit the properties of starlikeness and convexity, respectively (see also [27]). A harmonic mapping $f$ of the unit disk $\mathbb{D}$ is fully convex if it maps every circle $|z|=r<1$ in a one-to-one manner onto a convex curve. Such a harmonic mapping $f$ with $f(0)=0$ is fully starlike if it maps every 
circle $|z|=r<1$ in a one-to-one manner onto a curve that bounds a domain starlike with respect to the origin. Applying Theorem 2.2(iv) and using the fact that stable starlike (resp. stable convex) mappings are fully starlike (resp. fully convex) (see $[15,27])$, we have:

Corollary 2.4. Suppose that $\mathcal{G} \subset \mathcal{S}$ and $\mathcal{G} \triangleright \mathcal{G}_{H}^{0}$. If $\mathcal{G} \subset \mathcal{S}^{*}($ resp. $\mathcal{G} \subset \mathcal{K})$, then members of $\mathcal{G}_{H}^{0}$ are fully starlike (resp. fully convex) in $\mathbb{D}$.

The harmonic Koebe function

(4) $K(z)=H(z)+\overline{G(z)}, \quad H(z):=\frac{z-\frac{1}{2} z^{2}+\frac{1}{6} z^{3}}{(1-z)^{3}}, \quad G(z):=\frac{\frac{1}{2} z^{2}+\frac{1}{6} z^{3}}{(1-z)^{3}}$

shows that the classes $\mathcal{S}_{H}^{0}, \mathcal{S}_{H}^{* 0}$ and $\mathcal{C}_{H}^{0}$ are not harmonic analogues of any subfamily of $\mathcal{S}$ since

$$
H(z)+G(z)=\frac{z+\frac{1}{3} z^{3}}{(1-z)^{3}}, \quad z \in \mathbb{D},
$$

and $(H+G)(i / \sqrt{3})=(H+G)(-i / \sqrt{3})$ which imply that $H+G$ is not univalent in $\mathbb{D}$. Similarly, $\mathcal{K}_{H}^{0}$ is not a harmonic analogue of any subfamily $\mathcal{G} \subset \mathcal{S}$. For if $\mathcal{G} \triangleright \mathcal{K}_{H}^{0}$, then $\mathcal{G} \subset \mathcal{K}$. The harmonic half-plane mapping

$$
L(z)=M(z)+\overline{N(z)}, \quad M(z):=\frac{z-\frac{1}{2} z^{2}}{(1-z)^{2}}, \quad N(z):=\frac{-\frac{1}{2} z^{2}}{(1-z)^{2}}
$$

belongs to $\mathcal{K}_{H}^{0}$ and $M(z)-N(z)=z /(1-z)^{2} \notin \mathcal{K}$. These observations suggest that given a subfamily $\mathcal{G}_{H}^{0} \subset \mathcal{S}_{H}^{0}$, it is possible that $\mathcal{G}_{H}^{0}$ is not a harmonic analogue of any subclass of $\mathcal{S}$. This motivates us to determine a necessary and sufficient condition for a subfamily $\mathcal{G}_{H}^{0} \subset \mathcal{S}_{H}^{0}$ to be a harmonic analogue of some family $\mathcal{G} \subset \mathcal{S}$. This is contained in the following corollary.

Corollary 2.5. A subfamily $\mathcal{G}_{H}^{0} \subset \mathcal{S}_{H}^{0}$ is a harmonic analogue of some family $\mathcal{G} \subset \mathcal{S}$ if and only if $\mathcal{G}_{H}^{0} \subset \mathcal{S S}_{H}^{0}$.

Proof. The necessary part follows by Theorem 2.2(i). For the sufficient part, suppose that $\mathcal{G}_{H}^{0} \subset \mathcal{S S}_{H}^{0}$. Considering the set $\mathcal{G}=\left\{h+\epsilon g: h+\bar{g} \in \mathcal{G}_{H}^{0}\right.$ and $|\epsilon|=$ $1\}$, it is evident that $\mathcal{G} \subset \mathcal{S}$ and $\mathcal{G} \triangleright \mathcal{G}_{H}^{0}$ by using Lemma 2.1.

It is easy to see that if $\mathcal{I}$ and $\mathcal{J}$ are subclasses of $\mathcal{S}$ with $\mathcal{I} \triangleright \mathcal{I}_{H}^{0}$ and $\mathcal{J} \triangleright \mathcal{J}_{H}^{0}$, then $\mathcal{I} \cap \mathcal{J} \triangleright \mathcal{I}_{H}^{0} \cap \mathcal{J}_{H}^{0}$ and $\mathcal{I} \cup \mathcal{J} \triangleright \mathcal{I}_{H}^{0} \cup \mathcal{J}_{H}^{0}$. The next theorem determines the coefficient bounds for functions in the harmonic analogue $\mathcal{G}_{H}^{0}$.

Theorem 2.6. Suppose that $\mathcal{G} \subset \mathcal{S}$ and $\mathcal{G} \triangleright \mathcal{G}_{H}^{0}$. Let the Taylor coefficients $a_{n}(f)$ of the series of each $f \in \mathcal{G}$ satisfies $\left|a_{n}(f)\right| \leq p(n)$ for $n=2,3, \ldots$ where $p$ is a function of $n$. Then the respective Taylor coefficients $A_{n}(f)$ and $B_{n}(f)$ of the series of $h$ and $g$ of each function $f=h+\bar{g} \in \mathcal{G}_{H}^{0}$ satisfies

$$
\left|A_{n}(f)\right|+\left|B_{n}(f)\right| \leq p(n) \quad \text { for } \quad n=2,3, \ldots .
$$

In particular, we have 
(a) ||$A_{n}(f)|-| B_{n}(f)|| \leq p(n), \quad n=2,3, \ldots$.

(b) Let $h_{0} \in \mathcal{G}$ be such that its Taylor coefficients satisfy $\left|a_{n}\left(h_{0}\right)\right|=p(n)$ for $n=2,3, \ldots$ Then for an analytic function $g_{0}$, the harmonic function $f_{0}=h_{0}+\bar{g}_{0} \in \mathcal{G}_{H}^{0}$ if and only if $g_{0} \equiv 0$.

Proof. Let $f=h+\bar{g} \in \mathcal{G}_{H}^{0}$. Then $h+\epsilon g \in \mathcal{G}$ for each $|\epsilon|=1$ so that $\left|a_{n}(h+\epsilon g)\right| \leq p(n)$ for $n=2,3, \ldots$. But $a_{n}(h+\epsilon g)=A_{n}(f)+\epsilon B_{n}(f)$ for $n=2,3, \ldots$ so that (6) is satisfied with appropriate choice of $\epsilon=\epsilon(n)$.

The part (a) is evident from (6). For (b), suppose that $f_{0}=h_{0}+\bar{g}_{0} \in \mathcal{G}_{H}^{0}$. Then $\left|A_{n}\left(f_{0}\right)\right|+\left|B_{n}\left(f_{0}\right)\right| \leq p(n)$ for $n=2,3, \ldots$. But $\left|A_{n}\left(f_{0}\right)\right|=\left|a_{n}\left(h_{0}\right)\right|=$ $p(n)$ for $n=2,3, \ldots$ so that $B_{n}\left(f_{0}\right)=0$ for $n=2,3, \ldots$ Thus $g_{0} \equiv 0$. The converse part is obvious.

The next theorem determines the upper and lower bounds on the growth of a harmonic mapping in $\mathcal{G}_{H}^{0}$.

Theorem 2.7. Suppose that $\mathcal{G} \subset \mathcal{S}$ and $\mathcal{G} \triangleright \mathcal{G}_{H}^{0}$. If

$$
P(|z|) \leq\left|f^{\prime}(z)\right| \leq Q(|z|), \quad z \in \mathbb{D}
$$

for each $f \in \mathcal{G}$ where $P$ and $Q$ are integrable functions of $|z|$, then each $f \in \mathcal{G}_{H}^{0}$ satisfies

$$
\int_{0}^{|z|} P(\rho) d \rho \leq|f(z)| \leq \int_{0}^{|z|} Q(\rho) d \rho, \quad z \in \mathbb{D} .
$$

In particular, we have the following.

(i) The range of every function $f \in \mathcal{G}_{H}^{0}$ contains the disk

$$
\left\{w \in \mathbb{C}:|w|<\lim _{|z| \rightarrow 1} \int_{0}^{|z|} P(\rho) d \rho\right\}
$$

provided the limit exists.

(ii) The Jacobian $J_{f}$ of each function $f \in \mathcal{G}_{H}^{0}$ satisfies

$$
P^{2}(|z|) \leq J_{f}(z) \leq Q^{2}(|z|), \quad z \in \mathbb{D}
$$

Proof. Let $f=h+\bar{g} \in \mathcal{G}_{H}^{0}$. Then $h+\epsilon g \in \mathcal{G}$ for each $|\epsilon|=1$ so that

$$
P(|z|) \leq\left|h^{\prime}(z)+\epsilon g^{\prime}(z)\right| \leq Q(|z|), \quad z \in \mathbb{D} .
$$

In particular, this shows that

$$
P(|z|) \leq\left|h^{\prime}(z)\right|-\left|g^{\prime}(z)\right| \quad \text { and } \quad\left|h^{\prime}(z)\right|+\left|g^{\prime}(z)\right| \leq Q(|z|), \quad z \in \mathbb{D} .
$$

If $\Gamma$ is the radial segment from 0 to $z$, then

$$
|f(z)|=\left|\int_{\Gamma} \frac{\partial f}{\partial \zeta} d \zeta+\frac{\partial f}{\partial \bar{\zeta}} d \bar{\zeta}\right| \leq \int_{\Gamma}\left(\left|h^{\prime}(\zeta)\right|+\left|g^{\prime}(\zeta)\right|\right)|d \zeta| \leq \int_{0}^{|z|} Q(\rho) d \rho .
$$


Next, let $\Gamma$ be the pre-image under $f$ of the radial segment from 0 to $f(z)$. Then

$$
|f(z)|=\int_{\Gamma}\left|\frac{\partial f}{\partial \zeta} d \zeta+\frac{\partial f}{\partial \bar{\zeta}} d \bar{\zeta}\right| \geq \int_{\Gamma}\left(\left|h^{\prime}(\zeta)\right|-\left|g^{\prime}(\zeta)\right|\right)|d \zeta| \geq \int_{0}^{|z|} P(\rho) d \rho .
$$

This proves (7).

The covering result in (i) follows from the left hand inequality of (7) by letting $|z| \rightarrow 1$. For the proof of (ii), let $f=h+\bar{g} \in \mathcal{G}_{H}^{0}$. Then (8) gives $\left|h^{\prime}(z)\right|-$ $\left|g^{\prime}(z)\right| \leq Q(|z|)$ and $\left|h^{\prime}(z)\right|+\left|g^{\prime}(z)\right| \leq Q(|z|)$. Multiplying the corresponding sides of these two inequalities, we obtain $J_{f}(z) \leq Q^{2}(|z|)$ for $z \in \mathbb{D}$. The left hand inequality follows on similar lines.

If a subfamily $\mathcal{G} \subset \mathcal{S}$ is compact with respect to the topology of locally uniform convergence, then so is its harmonic analogue $\mathcal{G}_{H}^{0}$. This is seen by the following theorem.

Theorem 2.8. Suppose that $\mathcal{G} \subset \mathcal{S}$ and $\mathcal{G} \triangleright \mathcal{G}_{H}^{0}$. Then $\mathcal{G}$ is compact if and only if $\mathcal{G}_{H}^{0}$ is compact.

Proof. For necessary part, suppose that $f_{n}=h_{n}+\bar{g}_{n} \in \mathcal{G}_{H}^{0}$ for $n=1,2, \ldots$ and that $f_{n} \rightarrow f$ uniformly on compact subsets of $\mathbb{D}$. Then $f$ is harmonic and so $f=h+\bar{g}$. It is easy to see that $h_{n} \rightarrow h$ and $g_{n} \rightarrow g$ locally uniformly so that $h_{n}+\epsilon g_{n} \rightarrow h+\epsilon g$ for each $|\epsilon|=1$. Since $h_{n}+\epsilon g_{n} \in \mathcal{G}$, it follows that $h+\epsilon g \in \mathcal{G}$ for each $|\epsilon|=1$ using the compactness of $\mathcal{G}$. Thus $f=h+\bar{g} \in \mathcal{G}_{H}^{0}$.

For sufficient part, let $f_{n} \in \mathcal{G}$ for $n=1,2, \ldots$ such that $f_{n} \rightarrow f$ uniformly on compact subsets of $\mathbb{D}$. Then $f$ is univalent. Since $\mathcal{G} \subset \mathcal{G}_{H}^{0}$ and $\mathcal{G}_{H}^{0}$ is compact, $f \in \mathcal{G}_{H}^{0}$. By Theorem 2.2(ii), $f \in \mathcal{G}$.

The next theorem investigates the relation between the radius of starlikeness, convexity and close-to-convexity of the classes $\mathcal{G}$ and $\mathcal{G}_{H}^{0}$.

Theorem 2.9. Suppose that $\mathcal{G} \subset \mathcal{S}$ and $\mathcal{G} \triangleright \mathcal{G}_{H}^{0}$. Then the classes $\mathcal{G}$ and $\mathcal{G}_{H}^{0}$ have the same radius of starlikeness, convexity and close-to-convexity.

Proof. Since $\mathcal{G} \subset \mathcal{G}_{H}^{0}$, it suffices to show that if $r_{0}$ is the radius of starlikeness (resp. convexity and close-to-convexity) of $\mathcal{G}$, then $f$ is starlike (resp. convex and close-to-convex) in $|z|<r_{0}$ for each $f \in \mathcal{G}_{H}^{0}$. To see this, suppose that $f=h+\bar{g} \in \mathcal{G}_{H}^{0}$. Then the analytic functions $h+\epsilon g$ belong to the class $\mathcal{G}$. Consequently the functions $h+\epsilon g$ are starlike (resp. convex and close-toconvex) in $|z|<r_{0}$. In view of Lemma 2.1, it follows that $f$ is starlike (resp. convex and close-to-convex) in $|z|<r_{0}$.

For analytic functions

$$
f(z)=z+\sum_{n=2}^{\infty} a_{n} z^{n} \quad \text { and } \quad F(z)=z+\sum_{n=2}^{\infty} A_{n} z^{n}
$$


belonging to $\mathcal{A}$, their convolution (or Hadamard product) is defined as

$$
(f * F)(z)=z+\sum_{n=2}^{\infty} a_{n} A_{n} z^{n}, \quad z \in \mathbb{D} .
$$

In the harmonic case, with $f=h+\bar{g}$ and $F=H+\bar{G}$ belonging to $\mathcal{H}$, their harmonic convolution is defined as $f * F=h * H+\overline{g * G}$. Harmonic convolutions are investigated in $[7,8,9,12,33]$.

Suppose that $\mathcal{I}$ and $\mathcal{J}$ are subclasses of $\mathcal{H}$. We say that a class $\mathcal{I}$ is closed under convolution if $\mathcal{I} * \mathcal{I} \subset \mathcal{I}$, that is, if $f, g \in \mathcal{I}$ then $f * g \in \mathcal{I}$. Similarly, the class $\mathcal{I}$ is closed under convolution with members of $\mathcal{J}$ if $\mathcal{I} * \mathcal{J} \subset \mathcal{I}$. Given a subfamily $\mathcal{G} \subset \mathcal{S}$, the next theorem discusses the convolution properties of its harmonic analogue $\mathcal{G}_{H}^{0}$.

Theorem 2.10. Suppose that $\mathcal{G} \subset \mathcal{S}$ is closed under convolution and $\mathcal{G} \triangleright \mathcal{G}_{H}^{0}$. Then

(i) The convolution of each member of $\mathcal{G}_{H}^{0}$ with itself is again a member of $\mathcal{G}_{H}^{0}$;

(ii) If $(f+g) / 2 \in \mathcal{G}$ for all $f, g \in \mathcal{G}$, then $\mathcal{G}_{H}^{0}$ is closed under convolution.

Proof. Let $f=h+\bar{g} \in \mathcal{G}_{H}^{0}$. To prove (i), it suffices to show that $(h * h)+\epsilon(g * g) \in$ $\mathcal{G}$ for each $|\epsilon|=1$. For $|\epsilon|=1$, note that

$$
(h * h)+\epsilon(g * g)=(h+i \nu g) *(h-i \nu g)
$$

where $\pm \nu$ are square roots of $\epsilon$. Since $\mathcal{G}$ is closed under convolution, it follows that $(h * h)+\epsilon(g * g) \in \mathcal{G}$ so that $f * f \in \mathcal{G}_{H}^{0}$. This proves (i).

For the proof of (ii), let $f_{i}=h_{i}+\bar{g}_{i} \in \mathcal{G}_{H}^{0}(i=1,2)$. Considering the analytic functions

$$
F_{1}=\left(h_{1}-g_{1}\right) *\left(h_{2}-\epsilon g_{2}\right)=\left(h_{1} * h_{2}\right)-\epsilon\left(h_{1} * g_{2}\right)-\left(h_{2} * g_{1}\right)+\epsilon\left(g_{1} * g_{2}\right)
$$

and

$$
F_{2}=\left(h_{1}+g_{1}\right) *\left(h_{2}+\epsilon g_{2}\right)=\left(h_{1} * h_{2}\right)+\epsilon\left(h_{1} * g_{2}\right)+\left(h_{2} * g_{1}\right)+\epsilon\left(g_{1} * g_{2}\right)
$$

for $|\epsilon|=1$, we see that

$$
\frac{1}{2}\left(F_{1}+F_{2}\right)=\left(h_{1} * h_{2}\right)+\epsilon\left(g_{1} * g_{2}\right) .
$$

Since $F_{1}, F_{2} \in \mathcal{G}$ and using the hypothesis, it is easy to deduce that $f_{1} * f_{2} \in$ $\mathcal{G}_{H}^{0}$.

If $\mathcal{G}$ is a convex subset of $\mathcal{S}$, then $(1-t) f+t g \in \mathcal{G}$ for all $f, g \in \mathcal{G}$ and $t \in[0,1]$. As a result, Theorem 2.10(ii) gives the following corollary.

Corollary 2.11. Suppose that $\mathcal{G} \subset \mathcal{S}$ is a convex set and is closed under convolution. If $\mathcal{G} \triangleright \mathcal{G}_{H}^{0}$, then $\mathcal{G}_{H}^{0}$ is closed under convolution. 
In [12], Goodloe considered the Hadamard product $\tilde{*}$ of a harmonic function with an analytic function defined as follows:

$$
f \tilde{*} \varphi=\varphi \tilde{*} f=h * \varphi+\overline{g * \varphi},
$$

where $f=h+\bar{g}$ is harmonic and $\varphi$ is analytic in $\mathbb{D}$. The next theorem investigates the properties of the product $\tilde{*}$.

Theorem 2.12. Suppose that $\mathcal{G} \subset \mathcal{S}$ and $\mathcal{G} \triangleright \mathcal{G}_{H}^{0}$. Let $\mathcal{O}$ be a subfamily of $\mathcal{A}$ such that $\mathcal{G}$ is closed under convolution with members of $\mathcal{O}$. Then $\varphi \tilde{*} f \in \mathcal{G}_{H}^{0}$ for all $\varphi \in \mathcal{O}$ and $f \in \mathcal{G}_{H}^{0}$.

Proof. Let $f=h+\bar{g} \in \mathcal{G}_{H}^{0}$ and $\varphi \in \mathcal{O}$. Then

$$
\varphi \tilde{*} f=\varphi * h+\overline{\varphi * g}=H+\bar{G},
$$

where $H=\varphi * h$ and $G=\varphi * g$ are analytic in $\mathbb{D}$. Setting $F=H+\epsilon G=$ $\varphi *(h+\epsilon g)$ where $|\epsilon|=1$, we note that $F \in \mathcal{G}$ since $\mathcal{G} * \mathcal{O} \subset \mathcal{G}$. Thus $H+\bar{G} \in \mathcal{G}_{H}^{0}$ as desired.

The next theorem indicates that the classes $\mathcal{G}$ and $\mathcal{G}_{H}^{0}$ have similar convex combination properties.

Theorem 2.13. Suppose that $\mathcal{G} \subset \mathcal{S}$ and $\mathcal{G} \triangleright \mathcal{G}_{H}^{0}$. Then $\mathcal{G}$ is closed under convex combinations if and only if $\mathcal{G}_{H}^{0}$ is closed under convex combinations.

Proof. Firstly we will prove the necessary part. For $n=1,2, \ldots$, suppose that $f_{n} \in \mathcal{G}_{H}^{0}$ where $f_{n}=h_{n}+\bar{g}_{n}$. For $\sum_{n=1}^{\infty} t_{n}=1,0 \leq t_{n} \leq 1$, the convex combination of $f_{n}$ 's may be written as

$$
f(z)=\sum_{n=1}^{\infty} t_{n} f_{n}(z)=h(z)+\overline{g(z)},
$$

where

$$
h(z)=\sum_{n=1}^{\infty} t_{n} h_{n}(z) \text { and } g(z)=\sum_{n=1}^{\infty} t_{n} g_{n}(z) .
$$

are analytic in $\mathbb{D}$ with $h(0)=g(0)=h^{\prime}(0)-1=g^{\prime}(0)=0$. For $|\epsilon|=1$, we have

$$
(h+\epsilon g)(z)=\sum_{n=1}^{\infty} t_{n}\left(h_{n}+\epsilon g_{n}\right)(z), \quad z \in \mathbb{D} .
$$

Since the class $\mathcal{G}$ is closed under convex combination and $h_{n}+\epsilon g_{n} \in \mathcal{G}$ for $n=1,2, \ldots$, it follows that $h+\epsilon g \in \mathcal{G}$. Thus $f=h+\bar{g} \in \mathcal{G}_{H}^{0}$. This proves the necessary part.

The sufficient part follows by using the fact that $\mathcal{G} \subset \mathcal{G}_{H}^{0}$ and applying Theorem 2.2(ii).

Theorem 2.13 immediately yields:

Corollary 2.14. Suppose that $\mathcal{G} \subset \mathcal{S}$ and $\mathcal{G} \triangleright \mathcal{G}_{H}^{0}$. Then $\mathcal{G}$ is a convex set if and only if $\mathcal{G}_{H}^{0}$ is a convex set. 
Keeping in mind that $\mathcal{S} \triangleright \mathcal{S S}_{H}^{0}, \mathcal{S}^{*} \triangleright \mathcal{S S}_{H}^{* 0}, \mathcal{K} \triangleright \mathcal{S K}_{H}^{0}$ and $\mathcal{C} \triangleright \mathcal{S C}_{H}^{0}$, we determine the coefficient estimates, growth results, convolution properties and sharp bound for radius of starlikeness, convexity and close-to-convexity for the classes $\mathcal{S S}_{H}^{0}, \mathcal{S S}_{H}^{* 0}, \mathcal{S} \mathcal{K}_{H}^{0}$ and $\mathcal{S C}_{H}^{0}$, using the results proved in this section. Note that parts (i) and (ii) of the following theorem have been independently proved in [15, Section 8].

Theorem 2.15. Let $f=h+\bar{g} \in \mathcal{S}_{H}^{0}$ where $h$ and $g$ are given by (1).

(i) (Coefficient estimates) If $f \in \mathcal{S S}_{H}^{0}, \mathcal{S S}_{H}^{* 0}$ or $\mathcal{S C}_{H}^{0}$, then the sharp inequality ||$a_{n}|-| b_{n}|| \leq n$ holds for $n=2,3, \ldots$ Equality occurs for the analytic Koebe function $k(z)=z /(1-z)^{2}$. In case, $f \in \mathcal{S K}_{H}^{0}$ then ||$a_{n}|-| b_{n}|| \leq 1$ for $n=2,3, \ldots$, with the equality occurring for the analytic half-plane mapping $l(z)=z /(1-z)$.

(ii) (Growth estimates and covering theorem) If $f \in \mathcal{S S}_{H}^{0}, \mathcal{S S}_{H}^{* 0}$ or $\mathcal{S C}_{H}^{0}$, then we have

$$
\frac{|z|}{(1+|z|)^{2}} \leq|f(z)| \leq \frac{|z|}{(1-|z|)^{2}}, \quad z \in \mathbb{D} .
$$

In particular, the range $f(\mathbb{D})$ contains the disk $|w|<1 / 4$. These results are sharp for the analytic Koebe function $k$. If $f \in \mathcal{S K}_{H}^{0}$, then

$$
\frac{|z|}{1+|z|} \leq|f(z)| \leq \frac{|z|}{1-|z|}, \quad z \in \mathbb{D}
$$

and therefore the range $f(\mathbb{D})$ contains the disk $|w|<1 / 2$. The analytic half plane mapping $l$ shows that these results are best possible.

(iii) (Compactness) The classes $\mathcal{S S}_{H}^{0}, \mathcal{S S}_{H}^{* 0}, \mathcal{S K}_{H}^{0}$ and $\mathcal{S C}_{H}^{0}$ are compact with respect to the topology of locally uniform convergence.

(iv) (Radii of starlikeness, convexity and close-to-convexity) Let $r_{S}\left(\mathcal{G}_{H}^{0}\right)$, $r_{C}\left(\mathcal{G}_{H}^{0}\right)$ and $r_{C C}\left(\mathcal{G}_{H}^{0}\right)$ denote the radius of starlikeness, convexity and close-to-convexity, respectively of a subclass $\mathcal{G}_{H}^{0} \subset \mathcal{S}_{H}^{0}$. Then

$$
\begin{gathered}
r_{S}\left(\mathcal{S S}_{H}^{* 0}\right)=r_{S}\left(\mathcal{S K}_{H}^{0}\right)=r_{C}\left(\mathcal{S K}_{H}^{0}\right)=r_{C C}\left(\mathcal{S S}_{H}^{* 0}\right)=r_{C C}\left(\mathcal{S K}_{H}^{0}\right)=r_{C C}\left(\mathcal{S C}_{H}^{0}\right)=1 ; \\
r_{C}\left(\mathcal{S S}_{H}^{0}\right)=r_{C}\left(\mathcal{S S}_{H}^{* 0}\right)=r_{C}\left(\mathcal{S C}_{H}^{0}\right)=2-\sqrt{3} \\
r_{S}\left(\mathcal{S S}_{H}^{0}\right)=\tanh (\pi / 4), \quad \text { and } \quad r_{S}\left(\mathcal{S C}_{H}^{0}\right)=4 \sqrt{2}-5 .
\end{gathered}
$$

For $r_{C C}\left(\mathcal{S S}_{H}^{0}\right)$, refer to [16].

(v) (Convolution properties)

(a) If $f \in \mathcal{S} \mathcal{K}_{H}^{0}$, then $f * f \in \mathcal{S} \mathcal{K}_{H}^{0}$.

(b) If $\varphi \in \mathcal{K}$ and $f \in \mathcal{S S}_{H}^{* 0}$ (resp. $f \in \mathcal{S K}_{H}^{0}$ and $f \in \mathcal{S C}_{H}^{0}$ ), then $f \tilde{*} \varphi \in \mathcal{S S}_{H}^{* 0}\left(\right.$ resp. $f \tilde{*} \varphi \in \mathcal{S K}_{H}^{0}$ and $\left.f \tilde{*} \varphi \in \mathcal{S C}_{H}^{0}\right)$.

(vi) If $f=h+\bar{g} \in \mathcal{S} \mathcal{K}_{H}^{0}$, then

$$
\operatorname{Re} \frac{h(z)}{z}>\frac{1}{2}+\left|\frac{g(z)}{z}\right|
$$

for all $z \in \mathbb{D}$. The analytic half plane mapping l shows that the constant $1 / 2$ is best possible. 
Proof. Making use of the well-known coefficient estimates and distortion theorems for functions in the class $\mathcal{S}$ (see [14]), parts (i) and (ii) follow by applying Theorems 2.6 and 2.7, respectively. Theorem 2.8 gives (iii), while (iv) follows by using [14, Chapter 13] and Theorem 2.9. Since $\mathcal{K} * \mathcal{S}^{*} \subset \mathcal{S}^{*}, \mathcal{K} * \mathcal{K} \subset \mathcal{K}$ and $\mathcal{K} * \mathcal{C} \subset \mathcal{C}$, the convolution properties are easy to deduce from Theorems 2.10(i) and 2.12. For (vi), let $f=h+\bar{g} \in \mathcal{S K}_{H}^{0}$. Then $h+\epsilon g \in \mathcal{K}$ for each $|\epsilon|=1$. By the well-known Marx Strohhäcker theorem [22, Theorem 2.6(a), p. 57], it follows that $\operatorname{Re}(h+\epsilon g)(z) / z>1 / 2$ for $z \in \mathbb{D}$. By picking $\epsilon$ wisely, we obtain the desired result.

We close this section with the following remark.

Remark 2.16. It is clear that the classes $\mathcal{S S}_{H}^{0}, \mathcal{S S}_{H}^{* 0}$ and $\mathcal{S C}_{H}^{0}$ are not closed under convolution. However, since $\mathcal{K} * \mathcal{K} \subset \mathcal{K}, \mathcal{K} \triangleright \mathcal{S} \mathcal{K}_{H}^{0}$ and $\mathcal{K}$ is a non-convex set, it is expected that $\mathcal{S} \mathcal{K}_{H}^{0}$ is also not closed under convolution in view of Corollary 2.11. It will be an interesting open problem to determine whether $\mathcal{S K}_{H}^{0}$ is closed under convolution.

\section{Harmonic analogues of subclasses of $\mathcal{S}$}

In this section, we will determine the harmonic analogues of certain subclasses of $\mathcal{S}$. Apart from results of Section 2, we will make use of the following two lemmas which are the generalization of Theorems 2.10 and 2.12. Their proof being similar are omitted.

Lemma 3.1. Let $\mathcal{I}$ and $\mathcal{J}$ be subfamilies of $\mathcal{S}$ such that $\mathcal{I} * \mathcal{I} \subset \mathcal{J}$. If $\mathcal{I}_{H}^{0}$ and $\mathcal{J}_{H}^{0}$ denote the harmonic analogues of $I$ and $J$, respectively, then

(a) If $f \in \mathcal{I}_{H}^{0}$, then $f * f \in \mathcal{J}_{H}^{0}$;

(b) If $(f+g) / 2 \in \mathcal{J}$ for all $f, g \in \mathcal{J}$, then $\mathcal{I}_{H}^{0} * \mathcal{I}_{H}^{0} \subset \mathcal{J}_{H}^{0}$.

Lemma 3.2. Suppose that $\mathcal{I}$ and $\mathcal{J}$ are subfamilies of $\mathcal{S}$. Let $\mathcal{O} \subset \mathcal{A}$ be such that $f * g \in \mathcal{J}$ for all $f \in \mathcal{I}$ and $g \in \mathcal{O}$. Then $\varphi \tilde{*} f \in \mathcal{J}_{H}^{0}$ for all $\varphi \in \mathcal{O}$ and $f \in \mathcal{I}_{H}^{0}$, where $\mathcal{I} \triangleright \mathcal{I}_{H}^{0}, \mathcal{J} \triangleright \mathcal{J}_{H}^{0}$ and $\tilde{*}$ is defined by (10).

\subsection{Class $\mathcal{R}$}

Denote by $\mathcal{R}$ the class consisting of functions $f \in \mathcal{A}$ which satisfy $\operatorname{Re} f^{\prime}(z)>$ 0 for $z \in \mathbb{D}$. By well-known Noshiro-Warschawski Theorem (see [14, Chapter 7, p. 88]), $\mathcal{R} \subset \mathcal{S}$. In [19], MacGregor investigated the properties of functions in the class $\mathcal{R}$. Also, it is easy to see that $\mathcal{R}$ is a compact family and is closed under convex combinations. However, the class $\mathcal{R}$ is not closed under convolutions. The analytic function

$$
f(z)=-z-2 \log (1-z)=z+\sum_{n=2}^{\infty} \frac{2}{n} z^{n}
$$

belongs to $\mathcal{R}$ but $f * f \notin \mathcal{R}$. The first theorem of this section determines the harmonic analogue of the class $\mathcal{R}$ and discusses its properties. 
Theorem 3.3. The harmonic analogue of $\mathcal{R}$ is the class $\mathcal{R}_{H}^{0}$ defined by

$$
\mathcal{R}_{H}^{0}=\left\{f=h+\bar{g} \in \mathcal{H}: \operatorname{Re} h^{\prime}(z)>\left|g^{\prime}(z)\right| \text { for all } z \in \mathbb{D}\right\} .
$$

In particular, $\mathcal{R}_{H}^{0} \subset \mathcal{S C}_{H}^{0}$. Moreover, we have the following:

(i) If $f=h+\bar{g} \in \mathcal{R}_{H}^{0}$ where $h$ and $g$ are given by (1), then $\left|a_{n}\right|+\left|b_{n}\right| \leq 2 / n$ for $n=2,3, \ldots$ Equality holds for the function $f$ given by (11).

(ii) Every function $f \in \mathcal{R}_{H}^{0}$ satisfies

$$
-|z|+2 \log (1+|z|) \leq|f(z)| \leq-|z|-2 \log (1-|z|), \quad z \in \mathbb{D},
$$

and hence the range of each function $f \in \mathcal{R}_{H}^{0}$ contains the disk $|w|<$ $2 \log 2-1$. These results are sharp for the function $f$ given by (11).

(iii) The class $\mathcal{R}_{H}^{0}$ is compact with respect to the topology of locally uniform convergence.

(iv) $r_{C}\left(\mathcal{R}_{H}^{0}\right)=\sqrt{2}-1$ and $r_{C C}\left(\mathcal{R}_{H}^{0}\right)=1$.

(v) If $\varphi \in \mathcal{K}$ and $f \in \mathcal{R}_{H}^{0}$, then $f \tilde{*} \varphi \in \mathcal{R}_{H}^{0}$. Also, if $f \in \mathcal{A}$ with $\operatorname{Re} \varphi(z) / z>1 / 2$ for $z \in \mathbb{D}$ and $f \in \mathcal{R}_{H}^{0}$, then $f \tilde{*} \varphi \in \mathcal{R}_{H}^{0}$.

(vi) The class $\mathcal{R}_{H}^{0}$ is closed under convex combinations of its members.

Proof. Suppose that $\mathcal{R} \triangleright \mathcal{G}_{H}^{0}$. If $f=h+\bar{g} \in \mathcal{G}_{H}^{0}$, then the inequality $\operatorname{Re}\left(h^{\prime}(z)+\right.$ $\left.\epsilon g^{\prime}(z)\right)>0$ holds for each $z \in \mathbb{D}$ and $|\epsilon|=1$. With appropriate choice of $\epsilon=\epsilon(z)$, it follows that

$$
\operatorname{Re} h^{\prime}(z)>\left|g^{\prime}(z)\right|, \quad z \in \mathbb{D}
$$

so that $f \in \mathcal{R}_{H}^{0}$. To prove the reverse inclusion, let $f=h+\bar{g} \in \mathcal{R}_{H}^{0}$. Then for $|\epsilon|=1$ we have

$$
\operatorname{Re}\left(h^{\prime}(z)+\epsilon g^{\prime}(z)\right) \geq \operatorname{Re} h^{\prime}(z)-\left|g^{\prime}(z)\right|>0, \quad z \in \mathbb{D}
$$

which imply that $h+\epsilon g \in \mathcal{R}$ and hence $f \in \mathcal{G}_{H}^{0}$. This shows that $\mathcal{R} \triangleright \mathcal{R}_{H}^{0}$.

Since $\mathcal{R} \subset \mathcal{C}, \mathcal{R}_{H}^{0} \subset \mathcal{S C}_{H}^{0}$ by Theorem 2.2(iv). In view of [19, Theorems 1 and 2, p. 533], the proof of parts (i), (ii) and (iv) follow by applying Theorems $2.6,2.7$ and 2.9 , respectively. Theorems 2.8 and 2.13 verify the validity of (iii) and (vi), respectively. Since $\mathcal{K} * \mathcal{R} \subset \mathcal{R}$ (by [2, Corollary 3.10]), Theorem 2.12 shows that $f \tilde{*} \varphi \in \mathcal{R}_{H}^{0}$ if $\varphi \in \mathcal{K}$ and $f \in \mathcal{R}_{H}^{0}$. For the proof of the other part of (v), it suffices to show that if $\varphi \in \mathcal{A}$ with $\operatorname{Re} \varphi(z) / z>1 / 2$ and $f \in \mathcal{R}$, then $f * \varphi \in \mathcal{R}$. To see this, note that $(f * \varphi)^{\prime}(z)=f^{\prime}(z) * \varphi(z) / z$ for $z \in \mathbb{D}$. By [37, Lemma 4, p. 146], it follows that $\operatorname{Re}(f * \varphi)^{\prime}>0$ so that $f * \varphi \in \mathcal{R}$. This concludes the proof of the theorem.

Note that Mocanu [23] independently proved that if $f$ is a harmonic mapping in a convex domain $\Omega$ such that $\operatorname{Re} f_{z}(z)>\left|f_{\bar{z}}(z)\right|$ for $z \in \Omega$, then $f$ is univalent and sense-preserving in $\Omega$ while Ponnusamy et al. [30] showed that members of $\mathcal{R}_{H}^{0}$ are close-to-convex in $\mathbb{D}$. 
Now we will determine the radius of convexity for a certain family of harmonic functions. For $G \in \mathcal{A}$, consider the family

$$
\mathcal{R}_{H}^{0}(G)=\left\{f=h+\bar{g} \in \mathcal{H}: \operatorname{Re} \frac{h^{\prime}(z)}{G^{\prime}(z)}>\left|\frac{g^{\prime}(z)}{G^{\prime}(z)}\right| \text { for all } z \in \mathbb{D}\right\} .
$$

If $G(z)=z$, then $\mathcal{R}_{H}^{0}(G)$ reduces to $\mathcal{R}_{H}^{0}$. In [29], it has been proved that if $G \in \mathcal{K}$, then $\mathcal{R}_{H}^{0}(G) \subset \mathcal{S C}_{H}^{0}$ (see also $[23,25]$ ). The next theorem determines the radius of convexity of the class $\mathcal{R}_{H}^{0}(G)$ for specific choices of the function $G$.

Theorem 3.4. Let $r_{C}$ denotes the radius of convexity of the class $\mathcal{R}_{H}^{0}(G)$ for $G \in \mathcal{A}$.

(i) If $G \in \mathcal{S}$, then $r_{C}=3-2 \sqrt{2}$;

(ii) If $G \in \mathcal{S}^{*}$, then $r_{C}=3-2 \sqrt{2}$;

(iii) If $G \in \mathcal{K}$, then $r_{C}=2-\sqrt{3}$;

(iv) If $G \in \mathcal{R}$, then $r_{C}=\sqrt{5}-2$;

(v) If $G \in \mathcal{A}$ with $\operatorname{Re} G^{\prime}(z)>1 / 2$, then $r_{C}=3-2 \sqrt{2}$.

Moreover, all these results are sharp.

Proof. Let $f=h+\bar{g} \in \mathcal{R}_{H}^{0}(G)$. Setting $F_{\epsilon}=h+\epsilon g$ for $|\epsilon|=1$, note that

$$
\operatorname{Re} \frac{F_{\epsilon}^{\prime}(z)}{G^{\prime}(z)}=\operatorname{Re}\left(\frac{h^{\prime}(z)}{G^{\prime}(z)}+\epsilon \frac{g^{\prime}(z)}{G^{\prime}(z)}\right) \geq \operatorname{Re} \frac{h^{\prime}(z)}{G^{\prime}(z)}-\left|\frac{g^{\prime}(z)}{G^{\prime}(z)}\right|>0, \quad z \in \mathbb{D} .
$$

If $G \in \mathcal{S}$, then $F_{\epsilon}$ is convex in $|z|<3-2 \sqrt{2}$ by [31, Theorem 1, p. 32] for each $|\epsilon|=1$. By Lemma 2.1, $f$ is convex in $|z|<3-2 \sqrt{2}$. This proves (i). The proof of the other parts is similar.

\subsection{Class $\mathcal{W}$}

In [5], Chichra introduced the class $\mathcal{W}$ of analytic functions $f \in \mathcal{A}$ which satisfy $\operatorname{Re}\left(f^{\prime}(z)+z f^{\prime \prime}(z)\right)>0$ for $z \in \mathbb{D}$. He proved that the members of $\mathcal{W}$ are univalent in $\mathbb{D}$ by showing that $\mathcal{W} \subset \mathcal{R}$. Later Singh and Singh [36] proved that $\mathcal{W} \subset \mathcal{S}^{*}$. The class $\mathcal{W}$ is compact and is closed under convex combination of its members. Similar to the proof of Theorem 3.3, it can be shown that the set

$$
\mathcal{W}_{H}^{0}=\left\{f=h+\bar{g} \in \mathcal{H}: \operatorname{Re}\left(h^{\prime}(z)+z h^{\prime \prime}(z)\right)>\left|g^{\prime}(z)+z g^{\prime \prime}(z)\right| \text { for all } z \in \mathbb{D}\right\} .
$$

is the harmonic analogue of $\mathcal{W}$. By Theorem 2.2(iv), $\mathcal{W}_{H}^{0} \subset \mathcal{R}_{H}^{0} \cap \mathcal{S S}_{H}^{* 0}$. In particular, the members of $\mathcal{W}_{H}^{0}$ are fully starlike in $\mathbb{D}$ by Corollary 2.4. To determine the coefficient and growth estimates for functions in the class $\mathcal{W}_{H}^{0}$, we need to prove the following simple lemma.

Lemma 3.5. If $f \in \mathcal{W}$ is given by (9), then $\left|a_{n}\right| \leq 2 / n^{2}$ for $n=2,3, \ldots$ and

$$
-1+\frac{2}{|z|} \log (1+|z|) \leq\left|f^{\prime}(z)\right| \leq-1-\frac{2}{|z|} \log (1-|z|), \quad z \in \mathbb{D} .
$$


The function

$$
f(z)=-z-2 \int_{0}^{|z|} \frac{1}{t} \log (1-t) d t=z+\sum_{n=2}^{\infty} \frac{2}{n^{2}} z^{n}
$$

shows that all these results are sharp.

Proof. Observe that $f \in \mathcal{W}$ if and only if $z f^{\prime} \in \mathcal{R}$. The proof now follows by applying [19, Theorem 1, p. 533].

Theorem 3.6. Let $f=h+\bar{g} \in \mathcal{W}_{H}^{0}$ where $h$ and $g$ are given by (1). Then $\left|a_{n}\right|+\left|b_{n}\right| \leq 2 / n^{2}$ for $n=2,3, \ldots$ and

$$
-|z|+2 \int_{0}^{|z|} \frac{1}{t} \log (1+t) d t \leq|f(z)| \leq-|z|-2 \int_{0}^{|z|} \frac{1}{t} \log (1-t) d t, \quad z \in \mathbb{D} .
$$

In particular, the range $f(\mathbb{D})$ contains the disk $|w|<\pi^{2} / 6-1$. All these results are sharp for the function $f$ given by (12). Moreover, the following statements regarding the class $\mathcal{W}_{H}^{0}$ hold.

(i) The class $\mathcal{W}_{H}^{0}$ is compact with respect to the topology of locally uniform convergence.

(ii) $r_{S}\left(\mathcal{W}_{H}^{0}\right)=1=r_{C C}\left(\mathcal{W}_{H}^{0}\right)$.

(iii) The class $\mathcal{W}_{H}^{0}$ is closed under convolutions.

(iv) (a) If $\varphi \in \mathcal{K}$ and $f \in \mathcal{W}_{H}^{0}$, then $\varphi \tilde{*} f \in \mathcal{W}_{H}^{0}$;

(b) If $\varphi \in \mathcal{A}$ with $\operatorname{Re} \varphi(z) / z>1 / 2$ and $f \in \mathcal{W}_{H}^{0}$, then $\varphi \tilde{*} f \in \mathcal{W}_{H}^{0}$;

(c) If $\varphi \in \mathcal{W}$ and $f \in \mathcal{W}_{H}^{0}$, then $\varphi \tilde{*} f \in \mathcal{W}_{H}^{0} \cap \mathcal{S} \mathcal{K}_{H}^{0}$.

(v) The class $\mathcal{W}_{H}^{0}$ is closed under convex combinations.

(vi) If $f=h+\bar{g} \in \mathcal{W}_{H}^{0}$, then

$$
\operatorname{Re} h^{\prime}(z)>-1+2 \log 2+\left|g^{\prime}(z)\right|
$$

for all $z \in \mathbb{D}$. The function $f$ given by (12) shows that the constant $-1+2 \log 2$ cannot be replaced by any larger one.

Proof. The growth and coefficient estimates for the class $\mathcal{W}_{H}^{0}$ follow by Lemma 3.5 . Since $\mathcal{W}$ is a convex set and closed under convolutions (see [37, Theorem 3 , p. 150]), the class $\mathcal{W}_{H}^{0}$ is closed under convolutions by Corollary 2.11. This proves (iii). Since $\mathcal{W}_{H}^{0} \subset \mathcal{S S}_{H}^{* 0}$, (ii) is obviously true. To prove (iv), note that $\mathcal{K} * \mathcal{W} \subset \mathcal{W}$ (by [2, Corollary 3.10]) and if $\varphi \in \mathcal{A}$ with $\operatorname{Re} \varphi(z) / z>1 / 2$ and $f \in \mathcal{W}$, then $f * \varphi \in \mathcal{W}$ (by [37, Theorem 3', p. 150]). These observations lead to (a) and (b) by applying Theorem 2.12. Since $\mathcal{W} * \mathcal{W} \subset \mathcal{W} \cap \mathcal{K}$ (by [37, Theorems 3 and 4]), part (c) follows by Lemma 3.2. Theorems 2.8 and 2.13 verify the validity of the parts (i) and (v), respectively. For the proof of (vi), let $f=h+\bar{g} \in \mathcal{W}_{H}^{0}$. Then $h+\epsilon g \in \mathcal{W}$ for each $|\epsilon|=1$. Consequently, $\operatorname{Re}(h+\epsilon g)^{\prime}>-1+2 \log 2$ in $\mathbb{D}$ by [37, Theorem 1(a), p. 146]. In particular, we obtain the required result. 
Remark 3.7. Since $\mathcal{W} * \mathcal{W} \subset \mathcal{K}$, the convolution of each member of $\mathcal{W}_{H}^{0}$ with itself is convex in $\mathbb{D}$ by Lemma $3.1(a)$. However, since $\mathcal{K}$ is a non-convex set, it is not known whether $\mathcal{W}_{H}^{0} * \mathcal{W}_{H}^{0} \subset \mathcal{S} \mathcal{K}_{H}^{0}$ in view of Lemma 3.1(b).

\subsection{Classes $\mathcal{U}$ and $\mathcal{V}$}

Let $\mathcal{U}$ and $\mathcal{V}$ be subclasses of $\mathcal{A}$ consisting of functions $f$ of the form (9) that satisfy

$$
\sum_{n=2}^{\infty} n\left|a_{n}\right| \leq 1 \text { and } \sum_{n=2}^{\infty} n^{2}\left|a_{n}\right| \leq 1
$$

respectively. Clearly $\mathcal{V} \subset \mathcal{U}$. In [13], Goodman proved that $\mathcal{U} \subset \mathcal{S}^{*}$ and $\mathcal{V} \subset \mathcal{K}$. It is easy to see that $\mathcal{U} \subset \mathcal{R}$ and $\mathcal{V} \subset \mathcal{W}$. In fact, if $f \in \mathcal{U}$ is given by (9), then

$$
\operatorname{Re} f^{\prime}(z)=1+\operatorname{Re} \sum_{n=2}^{\infty} n a_{n} z^{n-1}>1-\sum_{n=2}^{\infty} n\left|a_{n}\right|>0 .
$$

Similarly, if $f \in \mathcal{V}$ is given by (9), then

$$
\operatorname{Re}\left(f^{\prime}(z)+z f^{\prime \prime}(z)\right)=1+\operatorname{Re} \sum_{n=2}^{\infty} n^{2} a_{n} z^{n-1}>1-\sum_{n=2}^{\infty} n^{2}\left|a_{n}\right|>0 .
$$

The next theorem determines the harmonic analogue of the classes $\mathcal{U}$ and $\mathcal{V}$.

Theorem 3.8. The harmonic analogues of the classes $\mathcal{U}$ and $\mathcal{V}$ are given by

$$
\mathcal{U}_{H}^{0}=\left\{f(z)=z+\sum_{n=2}^{\infty} a_{n} z^{n}+\overline{\sum_{n=2}^{\infty} b_{n} z^{n}} \in \mathcal{H}: \sum_{n=2}^{\infty} n\left(\left|a_{n}\right|+\left|b_{n}\right|\right) \leq 1\right\}
$$

and

$$
\mathcal{V}_{H}^{0}=\left\{f(z)=z+\sum_{n=2}^{\infty} a_{n} z^{n}+\overline{\sum_{n=2}^{\infty} b_{n} z^{n}} \in \mathcal{H}: \sum_{n=2}^{\infty} n^{2}\left(\left|a_{n}\right|+\left|b_{n}\right|\right) \leq 1\right\},
$$

respectively.

Proof. Suppose that $\mathcal{U} \triangleright \mathcal{G}_{H}^{0}$. If $f=h+\bar{g} \in \mathcal{G}_{H}^{0}$ where $h$ and $g$ are given by (1), then $h+\epsilon g \in \mathcal{U}$ for each $|\epsilon|=1$ so that

$$
\sum_{n=2}^{\infty} n\left|a_{n}+\epsilon b_{n}\right| \leq 1
$$

On choosing $\epsilon=\epsilon(n)$ wisely we deduce that $f \in \mathcal{U}_{H}^{0}$. Conversely if $f=h+\bar{g} \epsilon$ $\mathcal{U}_{H}^{0}$ where $h$ and $g$ are given by (1), then for $|\epsilon|=1$ we have

$$
\sum_{n=2}^{\infty} n\left|a_{n}+\epsilon b_{n}\right| \leq \sum_{n=2}^{\infty} n\left(\left|a_{n}\right|+\left|b_{n}\right|\right) \leq 1
$$

so that $h+\epsilon g \in \mathcal{U}$ and hence $f \in \mathcal{G}_{H}^{0}$. Thus $\mathcal{U} \triangleright \mathcal{U}_{H}^{0}$. Similarly it can be shown that $\mathcal{V} \triangleright \mathcal{V}_{H}^{0}$ 
In view of Theorem 2.2(iv), $\mathcal{U}_{H}^{0} \subset \mathcal{S S}_{H}^{* 0}$ and $\mathcal{V}_{H}^{0} \subset \mathcal{S} \mathcal{K}_{H}^{0}$. In particular, the members of $\mathcal{U}_{H}^{0}$ (resp. $\mathcal{V}_{H}^{0}$ ) are fully starlike (resp. fully convex) in $\mathbb{D}$ by Corollary 2.4. Also since $\mathcal{U} \subset \mathcal{R}$ and $\mathcal{V} \subset \mathcal{W}$, therefore $\mathcal{U}_{H}^{0} \subset \mathcal{R}_{H}^{0}$ (see also [30, Corollary 1.4, p. 25]) and $\mathcal{V}_{H}^{0} \subset \mathcal{W}_{H}^{0}$. Using the results of [34] and applying the theorems of Section 2, we have

Corollary 3.9. Let $f=h+\bar{g} \in \mathcal{S}_{H}^{0}$ where $h$ and $g$ are given by (1).

(a) If $f \in \mathcal{U}_{H}^{0}$, then

$\left|a_{n}\right| \leq 1 / n, \quad\left|b_{n}\right| \leq 1 / n \quad$ and $\quad|| a_{n}|-| b_{n}|| \leq 1 / n \quad$ for $n=2,3, \ldots$.

Equality occurs for the functions $z+z^{n} / n$ and $z+\bar{z}^{n} / n$. If $f \in \mathcal{V}_{H}^{0}$, then the sharp inequalities

$\left|a_{n}\right| \leq 1 / n^{2}, \quad\left|b_{n}\right| \leq 1 / n^{2} \quad$ and $\quad|| a_{n}|-| b_{n}|| \leq 1 / n^{2} \quad$ for $n=2,3, \ldots$

hold with the equality occurring for the functions $z+z^{n} / n^{2}$ and $z+$ $\bar{z}^{n} / n^{2}$.

(b) If $f \in \mathcal{U}_{H}^{0}$, then

$$
|z|-\frac{1}{2}|z|^{2} \leq|f(z)| \leq|z|+\frac{1}{2}|z|^{2}, \quad z \in \mathbb{D} .
$$

In particular, the range $f(\mathbb{D})$ contains the disc $|w|<1 / 2$. If $f \in \mathcal{V}_{H}^{0}$, then

$$
|z|-\frac{1}{4}|z|^{2} \leq|f(z)| \leq|z|+\frac{1}{4}|z|^{2}, \quad z \in \mathbb{D},
$$

and therefore $f(\mathbb{D})$ contains the disk $|w|<3 / 4$.

(c) The classes $\mathcal{U}_{H}^{0}$ and $\mathcal{V}_{H}^{0}$ are compact with respect to the topology of locally uniform convergence.

(d) $r_{S}\left(\mathcal{U}_{H}^{0}\right)=r_{C C}\left(\mathcal{U}_{H}^{0}\right)=r_{S}\left(\mathcal{V}_{H}^{0}\right)=r_{C}\left(\mathcal{V}_{H}^{0}\right)=r_{C C}\left(\mathcal{V}_{H}^{0}\right)=1$ and $r_{C}\left(\mathcal{U}_{H}^{0}\right)$ $=1 / 2$.

(e) The classes $\mathcal{U}_{H}^{0}$ and $\mathcal{V}_{H}^{0}$ are closed under convex combinations.

Avci and Zlotkiewicz [3] investigated certain properties of the classes $\mathcal{U}_{H}^{0}$ and $\mathcal{V}_{H}^{0}$ (see also [35]). The next theorem investigates the convolution properties of the classes $\mathcal{U}_{H}^{0}$ and $\mathcal{V}_{H}^{0}$.

Theorem 3.10. The classes $\mathcal{U}_{H}^{0}$ and $\mathcal{V}_{H}^{0}$ are closed under convolutions. Moreover, we have

(i) $\mathcal{U}_{H}^{0} * \mathcal{U}_{H}^{0} \subset \mathcal{S} \mathcal{K}_{H}^{0}$;

(ii) If $\varphi \in \mathcal{K}$ and $f \in \mathcal{U}_{H}^{0}$, then $\varphi \tilde{*} f \in \mathcal{U}_{H}^{0}$;

(iii) If $\varphi \in \mathcal{K}$ and $f \in \mathcal{V}_{H}^{0}$, then $\varphi \tilde{*} f \in \mathcal{V}_{H}^{0}$.

Proof. The main crux of the proof relies on the observation that if $f \in \mathcal{V}$ is given by (9), then $\sum_{n=2}^{\infty} n^{2}\left|a_{n}\right|^{2} \leq 1$. Since $\mathcal{U}$ and $\mathcal{V}$ are convex sets, therefore it suffices to show that the classes $\mathcal{U}$ and $\mathcal{V}$ are closed under convolution in view of Corollary 2.11. Let $f, F \in \mathcal{V}$ be given by (9). Then

$$
\sum_{n=2}^{\infty} n^{2}\left|a_{n} A_{n}\right| \leq \frac{1}{2} \sum_{n=2}^{\infty} n^{2}\left|a_{n}\right|^{2}+\frac{1}{2} \sum_{n=2}^{\infty} n^{2}\left|A_{n}\right|^{2} \leq 1
$$


using the fact that the geometric mean is less than or equal to the arithmetic mean. This shows that $f * F \in \mathcal{V}$. The same calculation shows that if $f, F \in \mathcal{U}$, then $f * F \in \mathcal{V} \subset \mathcal{U}$.

The proof of part (i) follows by Lemma 3.1(b) since $\mathcal{U} * \mathcal{U} \subset \mathcal{V}, \mathcal{V}$ is a convex set and $\mathcal{V} \subset \mathcal{K}$. Since the classes $\mathcal{U}$ and $\mathcal{V}$ are closed under convolution with convex functions, (ii) and (iii) follows immediately from Theorem 2.12.

\subsection{Class $\mathcal{S}_{\mathbb{R}}$}

Let $\mathcal{S}_{\mathbb{R}}$ be the subclass of $\mathcal{S}$ consisting of functions $f$ of the form (9) whose coefficients $a_{n}$ are all real. The following theorem determines its harmonic analogue.

Theorem 3.11. The harmonic analogue of $\mathcal{S}_{\mathbb{R}}$ is itself.

Proof. Suppose that $\mathcal{S}_{\mathbb{R}} \triangleright \mathcal{G}_{H}^{0}$. Then $\mathcal{S}_{\mathbb{R}} \subset \mathcal{G}_{H}^{0}$. To prove the reverse inclusion, let $f=h+\bar{g} \in \mathcal{G}_{H}^{0}$ where $h$ and $g$ are given by (1). Then $h+\epsilon g \in \mathcal{S}_{\mathbb{R}}$ for each $|\epsilon|=1$ which imply that all the coefficients $a_{n}+\epsilon b_{n}$ are real for each $|\epsilon|=1$. But this is possible only if $a_{n}$ are real and $b_{n}=0$ for $n=2,3, \ldots$. Thus $g \equiv 0$ and $f \in \mathcal{S}_{\mathbb{R}}$. Hence $\mathcal{S}_{\mathbb{R}} \triangleright \mathcal{S}_{\mathbb{R}}$.

\section{Harmonic integral operators}

In the theory of analytic univalent functions, Alexander operator $\Lambda$ given by (2) and Libera operator $\Theta$ defined by (3) play a crucial role. In this section, we will introduce and investigate the properties of harmonic Alexander operator and harmonic Libera operator.

\subsection{Harmonic Alexander operator}

Definition 4.1. Define an integral operator $\Lambda_{H}^{+}: \mathcal{H} \rightarrow \mathcal{H}$ by

$$
\Lambda_{H}^{+}[f]=\Lambda[h]+\overline{\Lambda[g]}, \quad f=h+\bar{g} \in \mathcal{H},
$$

where $\Lambda$ is the Alexander operator defined by (2). We call $\Lambda_{H}^{+}$the positive harmonic Alexander operator.

Since $\Lambda$ is linear, therefore so is the operator $\Lambda_{H}^{+}$, that is, $\Lambda_{H}^{+}\left[f_{1}+f_{2}\right]=$ $\Lambda_{H}^{+}\left[f_{1}\right]+\Lambda_{H}^{+}\left[f_{2}\right]$ for all $f_{1}, f_{2} \in \mathcal{H}$. The first theorem shows that if a subfamily $\mathcal{G} \subset \mathcal{S}$ is preserved under $\Lambda$, then its harmonic analogue $\mathcal{G}_{H}^{0}$ is preserved under $\Lambda_{H}^{+}$.

Theorem 4.2. Let $\mathcal{I}$ and $\mathcal{J}$ be subfamilies of $\mathcal{S}$ such that $\Lambda[\mathcal{I}] \subset \mathcal{J}$. Then $\Lambda_{H}^{+}\left[\mathcal{I}_{H}^{0}\right] \subset \mathcal{J}_{H}^{0}$ where $\mathcal{I} \triangleright \mathcal{I}_{H}^{0}$ and $\mathcal{J} \triangleright \mathcal{J}_{H}^{0}$.

Proof. Let $f=h+\bar{g} \in \mathcal{I}_{H}^{0}$. Since $\Lambda_{H}^{+}[f]=\Lambda[h]+\overline{\Lambda[g]}$ and $\mathcal{J} \triangleright \mathcal{J}_{H}^{0}$, it suffices to show that $\Lambda[h]+\epsilon \Lambda[g] \in \mathcal{J}$ for each $|\epsilon|=1$. But $\Lambda[h]+\epsilon \Lambda[g]=\Lambda[h+\epsilon g] \epsilon$ $\Lambda[\mathcal{I}] \subset \mathcal{J}$ since $\mathcal{I} \triangleright \mathcal{I}_{H}^{0}$.

Note that $\mathcal{R}_{H}^{0} \not \subset \mathcal{S}_{H}^{* 0}$ and $\mathcal{U}_{H}^{0} \not \subset \mathcal{K}_{H}^{0}$. Since $\Lambda[\mathcal{R}] \subset \mathcal{W} \subset \mathcal{S}^{*}$ and $\Lambda[\mathcal{U}] \subset \mathcal{V} \subset$ $\mathcal{K}$, Theorem 4.2 gives the following two corollaries. 


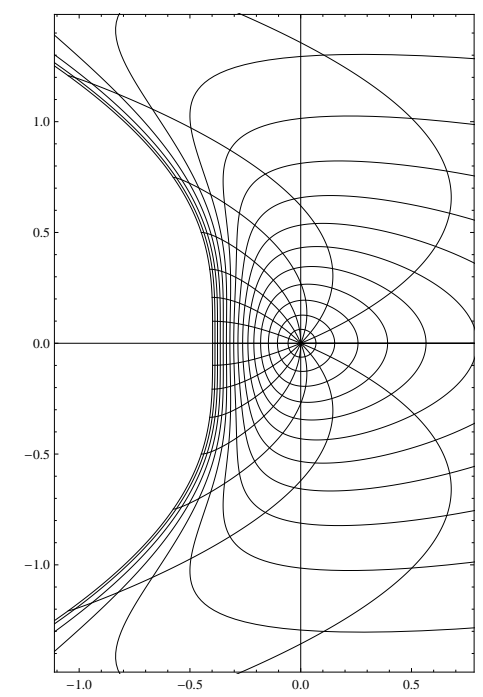

Figure 1. Graph of the function $\Lambda_{H}^{+}[K]$.

Corollary 4.3. $\Lambda_{H}^{+}\left[\mathcal{R}_{H}^{0}\right] \subset \mathcal{S} \mathcal{S}_{H}^{* 0}$ and $\Lambda_{H}^{+}\left[\mathcal{U}_{H}^{0}\right] \subset \mathcal{S} \mathcal{K}_{H}^{0}$.

Corollary 4.4. The classes $\mathcal{R}_{H}^{0}, \mathcal{W}_{H}^{0}, \mathcal{U}_{H}^{0}$ and $\mathcal{V}_{H}^{0}$ are preserved under $\Lambda_{H}^{+}$.

The Alexander operator $\Lambda$ provides a one-to-one correspondence between the classes $\mathcal{S}^{*}$ and $\mathcal{K}: f \in \mathcal{S}^{*}$ if and only if $\Lambda[f] \in \mathcal{K}$. A similar result holds for the positive harmonic Alexander operator which provides a one-toone correspondence between the classes $\mathcal{S S}_{H}^{* 0}$ and $\mathcal{S K}_{H}^{0}: f \in \mathcal{S S}_{H}^{* 0}$ if and only if $\Lambda_{H}^{+}[f] \in \mathcal{S K}_{H}^{0}$. In particular, the classes $\mathcal{S S}_{H}^{* 0}$ and $\mathcal{S K}_{H}^{0}$ are preserved under $\Lambda_{H}^{+}$. In fact, the class $\mathcal{S C}_{H}^{0}$ is also preserved under $\Lambda_{H}^{+}$since $\Lambda[\mathcal{C}] \subset \mathcal{C}$, a result proved by Merkes and Wright [21].

Gao [11] proved that if $f \in \mathcal{R}$, then $\operatorname{Re}(\Lambda[f](z) / z)>\left(\pi^{2} / 6\right)-1 \approx 0.6449$ $(z \in \mathbb{D})$ and the function $f$ given by (11) shows that the constant $\left(\pi^{2} / 6\right)-1$ cannot be replaced by any larger one. He also showed that if $f \in \mathcal{A}$ and $\operatorname{Re} f^{\prime}(z)>\left(6-\pi^{2}\right) /\left(24-\pi^{2}\right) \approx-0.2738$, then $\Lambda[f] \in \mathcal{S}^{*}$. These results are generalized in context of positive harmonic Alexander operator.

Theorem 4.5. Let $f=h+\bar{g} \in \mathcal{H}$.

(i) If $f \in \mathcal{R}_{H}^{0}$, then

$$
\operatorname{Re} \frac{\Lambda[h](z)}{z}>\left|\frac{\Lambda[g](z)}{z}\right|+\frac{\pi^{2}}{6}-1 \quad \text { for all } z \in \mathbb{D} .
$$

(ii) If $\operatorname{Re} h^{\prime}(z)>\left|g^{\prime}(z)\right|+\left(6-\pi^{2}\right) /\left(24-\pi^{2}\right)$ for all $z \in \mathbb{D}$, then $\Lambda_{H}^{+}[f] \in$ $\mathcal{S S}_{H}^{* 0}$. 
Proof. Since $\mathcal{R} \triangleright \mathcal{R}_{H}^{0}$, it follows that $h+\epsilon g \in \mathcal{R}$ for each $|\epsilon|=1$. Consequently

$$
\operatorname{Re}\left(\frac{\Lambda[h](z)}{z}+\epsilon \frac{\Lambda[g](z)}{z}\right)=\operatorname{Re} \frac{\Lambda[h+\epsilon g](z)}{z}>\frac{\pi^{2}}{6}-1
$$

for each $z \in \mathbb{D}$ and $|\epsilon|=1$. With appropriate choice of $\epsilon=\epsilon(z)$, we obtain (i).

For the proof of (ii), it is easy to see that $(h+\epsilon g)^{\prime}>\left(6-\pi^{2}\right) /\left(24-\pi^{2}\right)$ in $\mathbb{D}$ for each $|\epsilon|=1$. Hence $\Lambda[h+\epsilon g] \in \mathcal{S}^{*}$, or equivalently $\Lambda_{H}^{+}[f] \in \mathcal{S S}_{H}^{* 0}$.

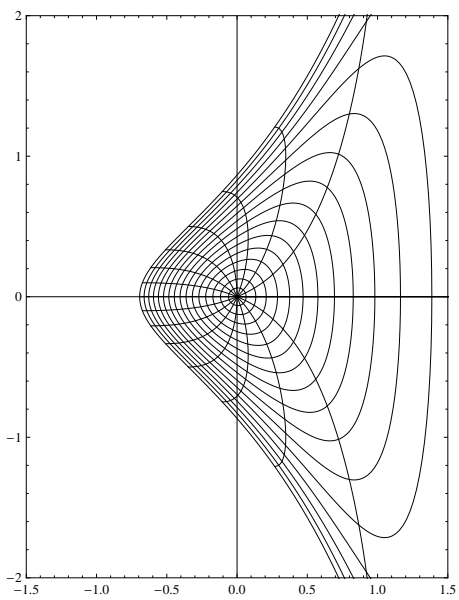

Figure 2. Graph of the function $\Lambda_{H}^{+}[L]$.

As discussed earlier, we have the inclusion $\Lambda_{H}^{+}\left[\mathcal{S S}_{H}^{* 0}\right] \subset \mathcal{S K}_{H}^{0}$. However, the inclusion $\Lambda_{H}^{+}\left[\mathcal{S}_{H}^{* 0}\right] \subset \mathcal{K}_{H}^{0}$ is not valid. To see this, note that the harmonic Koebe function $K$ given by (4) belongs to $\mathcal{S}_{H}^{* 0}$ and

$$
\begin{aligned}
\Lambda_{H}^{+}[K](z) & =\frac{1}{6}\left[\frac{z(5-3 z)}{(1-z)^{2}}-\log (1-z)\right]+\frac{1}{6}\left[\frac{z(3 z-1)}{(1-z)^{2}}-\log (1-z)\right] \\
& =\frac{2}{3} \frac{z}{(1-z)^{2}}+\frac{1}{3} i \operatorname{Im} \frac{z-3 z^{2}}{(1-z)^{2}}-\frac{1}{3} \log |1-z|, \quad z \in \mathbb{D} .
\end{aligned}
$$

The graph of the function $\Lambda_{H}^{+}[K]$ (see Figure 1) shows that the image domain is not even starlike. In particular, $\Lambda_{H}^{+}\left[\mathcal{S}_{H}^{* 0}\right] \not \subset \mathcal{S}_{H}^{* 0}$. Similarly, it can be shown that $\Lambda_{H}^{+}\left[\mathcal{K}_{H}^{0}\right] \not \subset \mathcal{K}_{H}^{0}$ by considering the harmonic half-plane mapping $L$ given by (5). Note that

$$
\begin{aligned}
\Lambda_{H}^{+}[L](z) & =\frac{1}{2}\left[-\log (1-z)+\frac{z}{1-z}\right]+\overline{\frac{1}{2}\left[-\log (1-z)-\frac{z}{1-z}\right]} \\
& =-\log |1-z|+i \operatorname{Im}\left(\frac{z}{1-z}\right) .
\end{aligned}
$$


Clearly Figure 2 depicts that the image domain $\Lambda_{H}^{+}[L](\mathbb{D})$ is not convex.

Although the members of $\Lambda_{H}^{+}\left[\mathcal{K}_{H}^{0}\right]$ need not map $\mathbb{D}$ onto a convex domain, its members are necessarily univalent and close-to-convex in $\mathbb{D}$ as seen by the following theorem.

Theorem 4.6. $\Lambda_{H}^{+}\left[\mathcal{K}_{H}^{0}\right] \subset \mathcal{S C}_{H}^{0}$.

Proof. Let $f=h+\bar{g} \in \mathcal{K}_{H}^{0}$. Then $h+\epsilon g \in \mathcal{C}$ for each $|\epsilon|=1$ by [7, Theorem 5.7 , p. 15]. Consequently $\Lambda[h]+\epsilon \Lambda[g]=\Lambda[h+\epsilon g] \in \mathcal{C}$ for each $|\epsilon|=1$, as $\Lambda[\mathcal{C}] \subset \mathcal{C}$. Since $\mathcal{C} \triangleright \mathcal{S C}_{H}^{0}$, we have $\Lambda_{H}^{+}[f] \in \mathcal{S C}_{H}^{0}$.

By Theorem 4.6, $\Lambda_{H}^{+}[L]$ is univalent and maps $\mathbb{D}$ onto a close-to-convex domain. Using the technique of shear construction [7, Theorem 5.3, p. 14] and convolution of harmonic mappings, the authors [28] have further investigated certain properties of positive harmonic Alexander operator.

The failure of the implication $\Lambda_{H}^{+}\left[\mathcal{S}_{H}^{* 0}\right] \subset \mathcal{K}_{H}^{0}$ motivates to introduce the following definition.

Definition 4.7. Define another integral operator $\Lambda_{H}^{-}: \mathcal{H} \rightarrow \mathcal{H}$ by

$$
\Lambda_{H}^{-}[f]=\Lambda[h]-\overline{\Lambda[g]}, \quad f=h+\bar{g} \in \mathcal{H},
$$

where $\Lambda$ is given by (2). We call $\Lambda_{H}^{-}$the negative harmonic Alexander operator.

By [10, Lemma, p. 108], it follows that $\Lambda_{H}^{-}\left[\mathcal{S}_{H}^{* 0}\right] \subset \mathcal{K}_{H}^{0}$. In particular, the classes $\mathcal{S}_{H}^{* 0}$ and $\mathcal{K}_{H}^{0}$ are preserved under the operator $\Lambda_{H}^{-}$. Therefore the mappings

$$
\Lambda_{H}^{-}[K](z)=\frac{2}{3} \frac{z}{(1-z)^{2}}+\frac{1}{3} \operatorname{Re} \frac{z-3 z^{2}}{(1-z)^{2}}-\frac{1}{3} i \arg (1-z)
$$

and

$$
\Lambda_{H}^{-}[L](z)=\operatorname{Re}\left(\frac{z}{1-z}\right)-i \arg (1-z)
$$

belong to $\mathcal{K}_{H}^{0}$, where $K$ and $L$ are given by (4) and (5), respectively (see Figure $3)$.

It is worth to remark that Theorems $4.2,4.5$ and 4.6 continue to hold for the negative harmonic Alexander operator $\Lambda_{H}^{-}$.

\subsection{Harmonic Libera operator}

Similar to Definition 4.1, we introduce the notion of harmonic Libera operator as follows.

Definition 4.8. Define an integral operator $\Theta_{H}: \mathcal{H} \rightarrow \mathcal{H}$ by

$$
\Theta_{H}[f]=\Theta[h]+\overline{\Theta[g]}, \quad f=h+\bar{g} \in \mathcal{H},
$$

where $\Theta$ is the Libera operator defined by (3). We call $\Theta_{H}$ the harmonic Libera operator. 


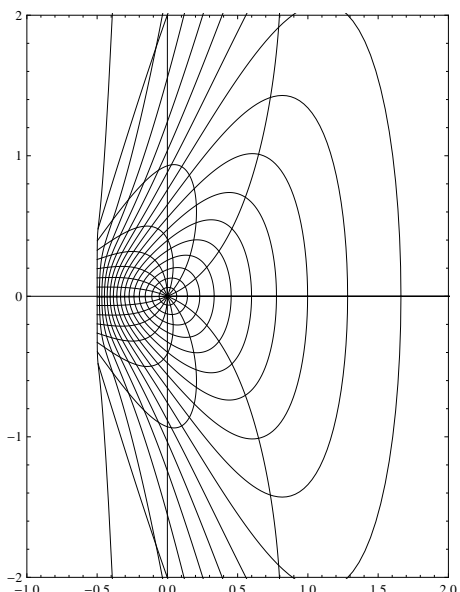

(A) $\Lambda_{H}^{-}[K]$

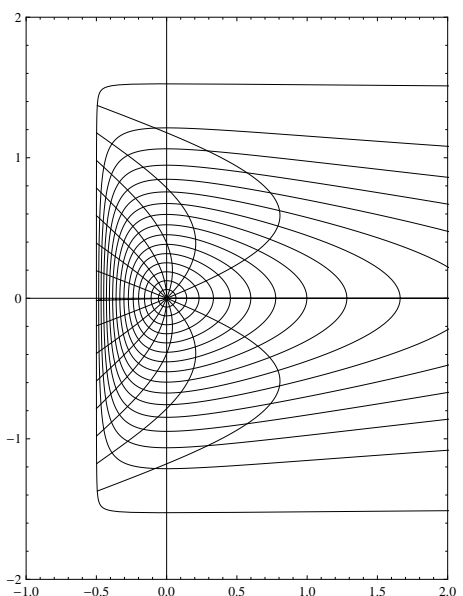

(в) $\Lambda_{H}^{-}[L]$

Figure 3. Images of the functions $K$ and $L$ under $\Lambda_{H}^{-}$.

The linearity of the operator $\Theta_{H}$ and the inclusions $\Theta\left[\mathcal{S}^{*}\right] \subset \mathcal{S}^{*}, \Theta[\mathcal{K}] \subset \mathcal{K}$, $\Theta[\mathcal{C}] \subset \mathcal{C}$ (see [18]) show that Theorems 4.2 and 4.6 hold for harmonic Libera operator $\Theta_{H}$ as well. Thus we obtain the following theorem.

Theorem 4.9. Let $\mathcal{I}$ and $\mathcal{J}$ be subfamilies of $\mathcal{S}$ such that $\Theta[\mathcal{I}] \subset \mathcal{J}$. Then $\Theta_{H}\left[\mathcal{I}_{H}^{0}\right] \subset \mathcal{J}_{H}^{0}$ where $\mathcal{I}_{H}^{0}$ and $\mathcal{J}_{H}^{0}$ are harmonic analogues of $\mathcal{I}$ and $\mathcal{J}$, respectively. In particular, the classes $\mathcal{S S}_{H}^{* 0}, \mathcal{S K}_{H}^{0}$ and $\mathcal{S C}_{H}^{0}$ are preserved under $\Theta_{H}$. Moreover, $\Theta_{H}\left[\mathcal{K}_{H}^{0}\right] \subset \mathcal{S C}_{H}^{0}$.

Mocanu [24] proved that $\Theta[\mathcal{R}] \subset \mathcal{S}^{*}$. Therefore, by Theorem 4.9, we have $\Theta_{H}\left[\mathcal{R}_{H}^{0}\right] \subset \mathcal{S S}_{H}^{* 0}$. Unlike positive harmonic Alexander operator (Corollary 4.3), the inclusion $\Theta_{H}\left[\mathcal{U}_{H}^{0}\right] \subset \mathcal{S} \mathcal{K}_{H}^{0}$ is not valid in general. This can be seen by considering the function $f_{0}(z)=z+\bar{z}^{2} / 2 \in \mathcal{U}_{H}^{0}$. Note that $\Theta_{H}\left[f_{0}\right](z)=z+\bar{z}^{2} / 3$ and the analytic function $z+z^{2} / 3 \notin \mathcal{K}$.

The classes $\mathcal{R}_{H}^{0}, \mathcal{W}_{H}^{0}, \mathcal{U}_{H}^{0}$ and $\mathcal{V}_{H}^{0}$ are also preserved under $\Theta_{H}$. This can be seen directly from Theorem 4.9 or by observing that we can write $\Theta_{H}[f]=f \tilde{*} \phi$ where $\tilde{*}$ is defined by $(10), \phi \in \mathcal{K}$ is given by

$$
\phi(z)=z+\sum_{n=2}^{\infty} \frac{2}{n+1} z^{n}=-2-\frac{2}{z} \log (1-z), \quad z \in \mathbb{D}
$$

and using the convolution results of these classes stated in Section 3. From the inclusion $\Theta_{H}\left[\mathcal{K}_{H}^{0}\right] \subset \mathcal{S C}_{H}^{0}$, it follows that the mapping

$$
\Theta_{H}[L](z)=\frac{z}{1-z}+\overline{\frac{z-2}{1-z}-\frac{2}{z} \log (1-z)}
$$




$$
=2 \operatorname{Re}\left(\frac{z}{1-z}\right)-2 \overline{\left(\frac{1}{1-z}+\frac{1}{z} \log (1-z)\right)}
$$

belongs to $\mathcal{S C}_{H}^{0}$, where $L \in \mathcal{K}_{H}^{0}$ is given by (5) (see Figure 4). However, $\Theta_{H}[L] \notin \mathcal{K}_{H}^{0}$, which shows that $\Theta_{H}\left[\mathcal{K}_{H}^{0}\right] \not \subset \mathcal{K}_{H}^{0}$.

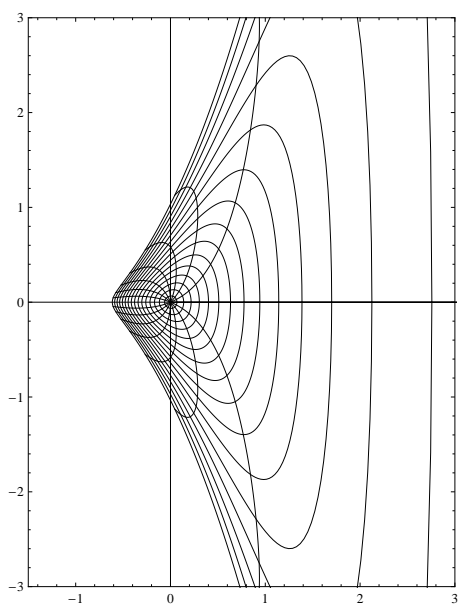

Figure 4. Graph of the function $\Theta_{H}[L]$.

Recall that a function $f \in \mathcal{H}$ is convex in the direction of the real (resp. imaginary) axis if the intersection of the image domain $f(\mathbb{D})$ with each horizontal (resp. vertical) line is connected. For further investigation of results regarding harmonic Libera operator, we need to prove the following theorem.

Theorem 4.10. Let $f=h+\bar{g} \in \mathcal{H}$ with $h(z)+g(z)=z /(1-z)$ and $\psi \in \mathcal{K}$. If

$$
\operatorname{Re}(1-z)^{2} h^{\prime}(z)>1 / 2 \quad \text { for } \quad z \in \mathbb{D},
$$

then $f \tilde{*} \psi \in \mathcal{S}_{H}^{0}$ and is convex in the direction of the imaginary axis, where $\tilde{*}$ is defined by $(10)$.

Proof. To apply [28, Lemma 1.1] to the function $f \tilde{*} \psi$, we need to show that $f \tilde{*} \psi$ is sense-preserving and $h * \psi+g * \psi$ is univalent and convex in the direction of imaginary axis. Since $h * \psi+g * \psi=(h+g) * \psi=z /(1-z) * \psi=\psi \in \mathcal{K}$, it only remains to show that the dilatation $w_{f \tilde{*} \psi}=(g * \psi)^{\prime} /(h * \psi)^{\prime}$ of $f \tilde{*} \psi$ satisfies $\left|w_{f \tilde{\kappa} \psi}\right|<1$ or equivalently $\operatorname{Re}\left(1-w_{f \tilde{*} \psi}\right) /\left(1+w_{f \tilde{*} \psi}\right)>0$ in $\mathbb{D}$. Using the identity $\psi=h * \psi+g * \psi$, it is easy to deduce that

$$
\operatorname{Re}\left(\frac{1-w_{f \tilde{*} \psi}}{1+w_{f \tilde{*} \psi}}\right)=\operatorname{Re} \frac{(h * \psi)^{\prime}-(g * \psi)^{\prime}}{(h * \psi)^{\prime}+(g * \psi)^{\prime}}=2 \operatorname{Re} \frac{(h * \psi)^{\prime}}{\psi^{\prime}}-1 .
$$


Since we can write

$$
\operatorname{Re} \frac{(h * \psi)^{\prime}}{\psi^{\prime}}=\operatorname{Re} \frac{\psi * \frac{z}{(1-z)^{2}}\left[(1-z)^{2} h^{\prime}(z)\right]}{\psi * \frac{z}{(1-z)^{2}}},
$$

where $\psi \in \mathcal{K}, z /(1-z)^{2} \in \mathcal{S}^{*}$ and $\operatorname{Re}(1-z)^{2} h^{\prime}(z)>1 / 2$, it follows that $\operatorname{Re}(h * \psi)^{\prime} / \psi^{\prime}>1 / 2$ for all $z \in \mathbb{D}$ by [32, Theorem 2.4, p. 54]. Hence (14) shows that the expression $\operatorname{Re}\left(1-w_{f \tilde{*} \psi}\right) /\left(1+w_{f \tilde{*} \psi}\right)$ is strictly positive in $\mathbb{D}$.

Since $\Theta_{H}[f]=f \tilde{*} \phi$ where $\phi \in \mathcal{K}$ is given by (13), Theorem 4.10 gives the following corollary.

Corollary 4.11. Let $f=h+\bar{g} \in \mathcal{H}$ with $h(z)+g(z)=z /(1-z)$ and $\operatorname{Re}(1-$ $z)^{2} h^{\prime}(z)>1 / 2$ for $z \in \mathbb{D}$. Then $\Theta_{H}[f] \in \mathcal{S}_{H}^{0}$ and is convex in the direction of the imaginary axis.

The harmonic half-plane mapping $L=M+\bar{N}$ given by (5) satisfies $M(z)+$ $N(z)=z /(1-z)$ and $\operatorname{Re}(1-z)^{2} M^{\prime}(z)=\operatorname{Re}(1 /(1-z))>1 / 2$, so $\Theta_{H}[L] \in \mathcal{S}_{H}^{0}$ and is convex in the direction of the imaginary axis (which is clearly evident from Figure 4) by Corollary 4.11. We give another example illustrating Corollary 4.11 .

Example 4.12. Consider the harmonic function $\Psi(z)=\psi_{1}+\bar{\psi}_{2} \in \mathcal{H}$ where

$$
\psi_{1}(z)=\frac{1}{4} \log \left(\frac{1+z}{1-z}\right)+\frac{1}{2} \frac{z}{1-z} \quad \text { and } \quad \psi_{2}(z)=-\frac{1}{4} \log \left(\frac{1+z}{1-z}\right)+\frac{1}{2} \frac{z}{1-z} .
$$

In fact, $\Psi \in \mathcal{K}_{H}^{0}$ and is constructed by shearing the conformal mapping $l(z)=$ $z /(1-z)$ in the direction of imaginary axis with dilatation $w_{\Psi}(z)=z$. Note that $\operatorname{Re}(1-z)^{2} \psi_{1}^{\prime}(z)=\operatorname{Re}(1 /(1+z))>1 / 2$ for $z \in \mathbb{D}$. Hence, by Corollary 4.11 , $\Theta_{H}[\Psi]=\Theta\left[\psi_{1}\right]+\overline{\Theta\left[\psi_{2}\right]} \in \mathcal{S}_{H}^{0}$ and is convex in the direction of the imaginary axis, where

$$
\Theta\left[\psi_{1}\right](z)=\frac{1}{2} \log \left(\frac{1+z}{1-z}\right)+\frac{1}{2 z} \log \left(1-z^{2}\right)-1-\frac{1}{z} \log (1-z)
$$

and

$$
\Theta\left[\psi_{2}\right](z)=-\frac{1}{2} \log \left(\frac{1+z}{1-z}\right)-\frac{1}{2 z} \log \left(1-z^{2}\right)-1-\frac{1}{z} \log (1-z) .
$$

The images of radial segments and concentric circles inside $\mathbb{D}$ under $\Psi$ and $\Theta_{H}[\Psi]$ are shown in Figure 5 .

Example 4.13. If $K=H+\bar{G} \in \mathcal{S}_{H}^{* 0}$ is the harmonic Koebe function given by (4), then

$\Theta_{H}[K]=\Theta[H]+\overline{\Theta[G]}=\frac{2}{3} \operatorname{Re}\left(\frac{3 z-1}{z(1-z)^{2}}+\frac{1}{z}-1\right)-\overline{\frac{2}{z}\left(\frac{z}{1-z}+\log (1-z)\right)}$. 


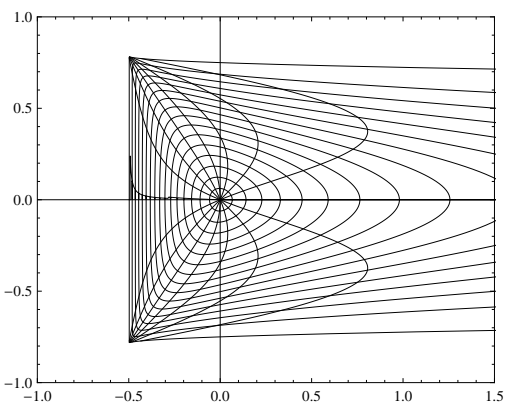

(A) $\Phi$

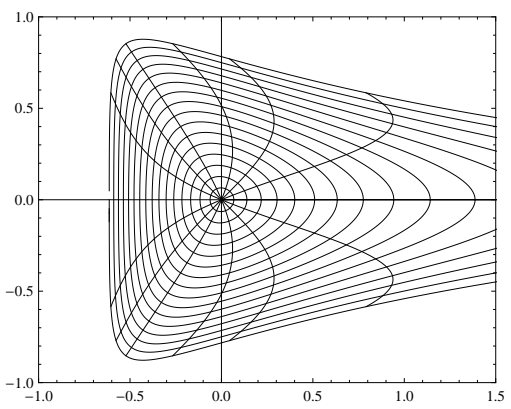

(B) $\Theta_{H}[\Psi]$

Figure 5. Images of the functions $\Psi$ and $\Theta_{H}[\Psi]$.

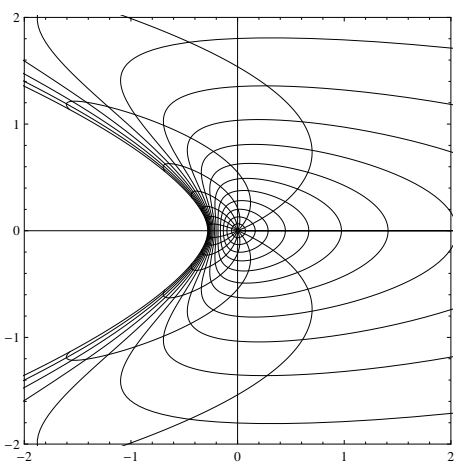

Figure 6. Graph of the function $\Theta_{H}[K]$.

Figure 6 clearly depicts that the image domain $\Theta_{H}[K](\mathbb{D})$ is not starlike. This shows that $\Theta_{H}\left[\mathcal{S}_{H}^{* 0}\right] \not \subset \mathcal{S}_{H}^{* 0}$.

However, it can be shown that $\Theta_{H}[K] \in \mathcal{S}_{H}^{0}$ and is convex in the direction of the real axis. To see this, note that $\Theta_{H}[K]=K \tilde{*} \phi$ where $\phi \in \mathcal{K}$ is given by (13). Since $H-G=z /(1-z)^{2}$ and $z \phi^{\prime} \in \mathcal{S}^{*}$ is convex in the direction of real axis (see Figure $7(\mathrm{~A})$ ), it follows that $H * \phi-G * \phi=z \phi^{\prime}$ is univalent and convex in the direction of real axis. Moreover, the dilatation $w_{K \tilde{*} \phi}=(G * \phi)^{\prime} /(H * \phi)^{\prime}$ of $K \tilde{*} \phi$ satisfies

$$
\operatorname{Re}\left(\frac{1+w_{K \tilde{*} \phi}}{1-w_{K \tilde{*} \phi}}\right)=2 \operatorname{Re} \frac{(H * \phi)^{\prime}}{\left(z \phi^{\prime}\right)^{\prime}}-1=2 \operatorname{Re} \frac{\phi * H * k}{\phi * k * k}-1
$$

which is clearly positive in $\mathbb{D}$ (the dashed line in Figure $7(\mathrm{~B})$ represents the line $\operatorname{Re} z=1 / 2)$, where $k(z)=z /(1-z)^{2}$ is the Koebe function. By [28, Lemma 1.1], $\Theta_{H}[K]=K \tilde{*} \phi \in \mathcal{S}_{H}^{0}$ and is convex in the direction of the real axis.

We close this section with the following remark. 


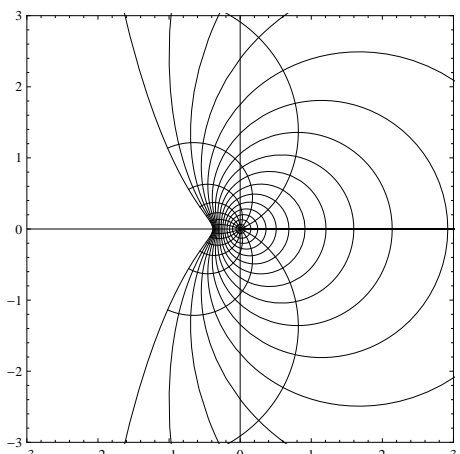

(A) $z \phi^{\prime}$

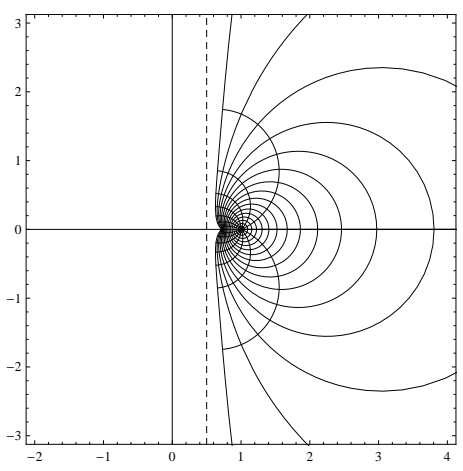

(в) $(\phi * H *$ $k) /(\phi * k * k)$

FiguRE 7. Mapping properties of the function $\phi$.

Remark 4.14. As discussed earlier, the classes $\mathcal{S}_{H}^{* 0}$ and $\mathcal{K}_{H}^{0}$ are not preserved under $\Theta_{H}$. Analogous to Definition 4.7, if we define another notion of harmonic Libera operator $\widetilde{\Theta}_{H}: \mathcal{H} \rightarrow \mathcal{H}$ by $\widetilde{\Theta}_{H}[f]=\Theta[h]-\bar{\Theta}[g]$ where $f=h+\bar{g} \in \mathcal{H}$ and $\Theta$ is the Libera operator defined by (3), then also $\widetilde{\Theta}_{H}\left[\mathcal{S}_{H}^{* 0}\right] \not \subset \mathcal{S}_{H}^{* 0}$ and $\widetilde{\Theta}_{H}\left[\mathcal{K}_{H}^{0}\right] \not \subset \mathcal{K}_{H}^{0}$. This can observed by Figure 8 which depicts the graph of the function

$$
\widetilde{\Theta}_{H}[L]=\Theta[M]-\overline{\Theta[N]}=2 i \operatorname{Im}\left(\frac{z}{1-z}\right)+2 \overline{\left(\frac{1}{1-z}+\frac{1}{z} \log (1-z)\right)}
$$

where $L$ is given by $(5)$. The image domain $\widetilde{\Theta}_{H}[L](\mathbb{D})$ is not even starlike.

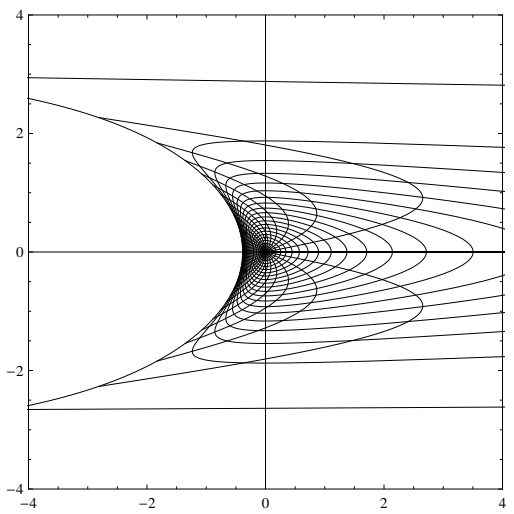

Figure 8. Graph of the function $\widetilde{\Theta}_{H}[L]$. 


\section{References}

[1] J. W. Alexander, Functions which map the interior of the unit circle upon simple regions, Ann. of Math. (2) 17 (1915), no. 1, 12-22.

[2] R. M. Ali, V. Ravichandran, and N. K. Jain, Convolution of certain analytic functions, J. Anal. 18 (2010), 1-8.

[3] Y. Avciand E. Złotkiewicz, On harmonic univalent mappings, Ann. Univ. Mariae CurieSkłodowska Sect. A 44 (1990), 1-7.

[4] D. M. Campbell and V. Singh, Valence properties of the solution of a differential equation, Pacific J. Math. 84 (1979), no. 1, 29-33.

[5] P. N. Chichra, New subclasses of the class of close-to-convex functions, Proc. Amer. Math. Soc. 62 (1976), no. 1, 37-43.

[6] M. Chuaqui, P. Duren, and B. Osgood, Curvature properties of planar harmonic mappings, Comput. Methods Funct. Theory 4 (2004), no. 1, 127-142.

[7] J. Clunie and T. Sheil-Small, Harmonic univalent functions, Ann. Acad. Sci. Fenn. Ser. A I Math. 9 (1984), 3-25.

[8] M. Dorff, Convolutions of planar harmonic convex mappings, Complex Variables Theory Appl. 45 (2001), no. 3, 263-271.

[9] M. Dorff, M. Nowak, and M. Wołoszkiewicz, Convolutions of harmonic convex mappings, Complex Var. Elliptic Equ. 57 (2012), no. 5, 489-503.

[10] P. Duren, Harmonic Mappings in the Plane, Cambridge Tracts in Mathematics, 156, Cambridge Univ. Press, Cambridge, 2004.

[11] C. Y. Gao, On the starlikeness of the Alexander integral operator, Proc. Japan Acad. Ser. A Math. Sci. 68 (1992), no. 10, 330-333.

[12] M. R. Goodloe, Hadamard products of convex harmonic mappings, Complex Var. Theory Appl. 47 (2002), no. 2, 81-92.

[13] A. W. Goodman, Univalent functions and nonanalytic curves, Proc. Amer. Math. Soc. 8 (1957), 598-601.

[14] _ Univalent Functions. Vol. I and II, Mariner, Tampa, FL, 1983.

[15] R. Hernández and M. J. Martín, Stable geometric properties of analytic and harmonic functions, Math. Proc. Cambridge Philos. Soc. 155 (2013), 343-359.

[16] J. Krzyż, The radius of close-to-convexivity within the family of univalent functions, Bull. Acad. Polon. Sci. Sér. Sci. Math. Astronom. Phys. 10 (1962), 201-204.

[17] J. Krzyż and Z. Lewandowski, On the integral of univalent functions, Bull. Acad. Polon. Sci. Sér. Sci. Math. Astronom. Phys. 11 (1963), 447-448.

[18] R. J. Libera, Some classes of regular univalent functions, Proc. Amer. Math. Soc. 16 (1965), 755-758.

[19] T. H. MacGregor, Functions whose derivative has a positive real part, Trans. Amer. Math. Soc. 104 (1962), 532-537.

[20] - A class of univalent functions, Proc. Amer. Math. Soc. 15 (1964), 311-317.

[21] E. P. Merkes and D. J. Wright, On the univalence of a certain integral, Proc. Amer. Math. Soc. 27 (1971), 97-100.

[22] S. S. Miller and P. T. Mocanu, Differential Subordinations, Monographs and Textbooks in Pure and Applied Mathematics, 225, Dekker, New York, 2000.

[23] P. T. Mocanu, Sufficient conditions of univalency for complex functions in the class $C^{1}$, Anal. Numér. Théor. Approx. 10 (1981), no. 1, 75-79.

[24] $153-155$.

[25] (2011), no. 3, 759-766.

[26] S. Nagpal and V. Ravichandran, A subclass of close-to-convex harmonic mappings, Complex Var. Elliptic Equ. 59 (2014), no. 2, 204-216. 
[27] - Fully starlike and fully convex harmonic mappings of order $\alpha$, Ann. Polon. Math. 108 (2013), no. 1, 85-107.

[28] _ Univalence and convexity in one direction of the convolution of harmonic mappings, Complex Var. Elliptic Equ. (2013), DOI:10.1080/17476933.2013.836188.

[29] S. Ponnusamy and A. Sairam Kaliraj, On harmonic close-to-convex functions, Comput. Methods Funct. Theory 12 (2012), no. 2, 669-685.

[30] S. Ponnusamy, H. Yamamoto, and H. Yanagihara, Variability regions for certain families of harmonic univalent mappings, Complex Var. Elliptic Equ. 58 (2013), no. 1, 23-34.

[31] J. S. Ratti, The radius of convexity of certain analytic functions, Indian J. Pure Appl. Math. 1 (1970), no. 1, 30-36.

[32] S. Ruscheweyh, Convolutions in Geometric Function Theory, Séminaire de Mathématiques Supérieures, 83, Presses Univ. Montréal, Montreal, QC, 1982.

[33] S. Ruscheweyh and L. C. Salinas, On the preservation of direction-convexity and the Goodman-Saff conjecture, Ann. Acad. Sci. Fenn. Ser. A I Math. 14 (1989), no. 1, 63-73.

[34] H. Silverman, Univalent functions with negative coefficients, Proc. Amer. Math. Soc. 51 (1975), 109-116.

[35] - Harmonic univalent functions with negative coefficients, J. Math. Anal. Appl. 220 (1998), no. 1, 283-289.

[36] R. Singh and S. Singh, Starlikeness and convexity of certain integrals, Ann. Univ. Mariae Curie-Skłodowska Sect. A 35 (1981), 145-148 (1984).

[37] Convolution properties of a class of starlike functions, Proc. Amer. Math. Soc. 106 (1989), no. 1, 145-152.

Sumit NAGPAL

Department of Mathematics

UNIVERSiTY OF DeLhi

DELHI-110 007, INDIA

E-mail address: sumitnagpal.du@gmail.com

V. RAVICHANDRAN

Department of Mathematics

UNIVERSITY OF DELHI

DelHI-110 007, InDIA

E-mail address: vravi68@gmail.com 